\title{
String solitons and T-duality
}

\section{Eric A. Bergshoeff ${ }^{a}$ and Fabio Riccioni ${ }^{b}$}

\author{
${ }^{a}$ Centre for Theoretical Physics, University of Groningen, \\ Nijenborgh 4, 9747 AG Groningen, The Netherlands \\ ${ }^{b}$ Department of Mathematics, Kings College London, \\ Strand London, WC2R 2LS, U.K.
}

E-mail: E.A.Bergshoeff@rug.nl, Fabio.Riccioni@kcl.ac.uk

ABSTRACT: We construct for arbitrary dimensions a universal T-duality covariant expression for the Wess-Zumino terms of supersymmetric String Solitons in toroidally compactified string theories with 32 supercharges. The worldvolume fields occurring in the effective action of these String Solitons form either a vector or a tensor multiplet with 16 supercharges. We determine the dimensions of the conjugacy classes under T-duality to which these String Solitons belong. We do this in two steps. First, we determine the T-duality representations of the $p$-forms of maximal supergravities that contain the potentials that couple to these String Solitons. We find that these are $p$-forms, with $D-4 \leq p \leq 6$ if $D \geq 6$ and with $D-4 \leq p \leq D$ if $D<6$, transforming in the antisymmetric representation of rank $m=p+4-D \leq 4$ of the T-duality symmetry $\mathrm{SO}(10-D, 10-D)$. All branes support vector multiplets except when $m=10-D$. In that case the T-duality representation splits, for $D<10$, into a selfdual and anti-selfdual part, corresponding to 5branes supporting either a vector or a tensor multiplet. In a second step we show that only certain well-defined lightlike directions in the anti-symmetric tensor representations of the T-duality group correspond to supersymmetric String Solitons. These lightlike directions define the conjugacy classes. As a by-product we show how by a straightforward procedure all solitonic fields of maximal supergravity are derived using the Kac-Moody algebra $E_{11}$.

KeYwords: p-branes, D-branes, String Duality

ARXIV EPRINT: 1102.0934 


\section{Contents}

1 Introduction $\quad 1$

2 Solitonic fields in $D<10$ dimensions $\quad 8$

3 String solitons in ten dimensions $\quad \mathbf{1 2}$

$\begin{array}{ll}3.1 \text { The IIB solitonic WZ terms } & 14\end{array}$

$\begin{array}{ll}3.2 & \text { The IIA solitonic WZ terms } \\ \end{array}$

4 Gauge algebra of solitonic fields in any dimension $\quad 17$

5 String solitons in any dimension $\quad 22$

$\begin{array}{llr}6 & \text { Central charges } & 28\end{array}$

$\begin{array}{lll}7 & \mathrm{E}_{11} \text { and a ten-dimensional origin } & 31\end{array}$

$7.1 \mathrm{E}_{11}$ and mixed-symmetry fields 31

$\begin{array}{lll}7.2 & \mathrm{E}_{11} \text { and T-duality } & 37\end{array}$

8 Conclusions $\quad 39$

$\begin{array}{ll}\text { A Spinor representations of } \mathrm{SO}(d, d) & 43\end{array}$

\section{Introduction}

Supergravity theories provide important information about the branes of string theory, of which they are the low-energy limit. The (bosonic) physical states of IIA and IIB supergravity are described by the metric and a set of $p$-form potentials, with $0 \leq p \leq 4$. These latter fields couple to (half-supersymmetric) electric $(p-1)$-branes whereas their Poincare duals couple to (half-supersymmetric) magnetic $(7-p)$-branes. ${ }^{1}$ It turns out that IIA and IIB supergravity can be extended with 9 -form and 10-form potentials that do not describe any physical degrees of freedom [1-4]. Nevertheless, the full (on-shell) supersymmetry algebra can be realised on these fields. It is therefore perfectly legitimate to add them to the supergravity theory and to see whether they couple to branes as well. Perhaps the best known example of such a field is the 9 -form potential of IIA supergravity [1]. Its equation of motion follows from a duality relation between the 10-form curvature of this potential and the Romans mass parameter $m$ [5]. This potential couples to the D8-brane,

\footnotetext{
${ }^{1}$ This excludes the IIA/IIB dilaton, but includes the axion of IIB supergravity which couples to the D-instanton, or "D(-1)-brane", whereas its dual RR 8-form potential couples to the D7-brane. The IIA/IIB dilatonic dual 8-form potential will be discussed in this paper.
} 
or domain-wall, of Type IIA string theory. One can also add a set of 10 -form potentials to IIA/IIB supergravity [2-4]. In the case of IIB supergravity one of these fields couples to the D9-brane. But there are more 10-form potentials on which the IIA/IIB supersymmetry algebra can be realised. Whether these other potentials couple to branes as well is less clear. Recently, the U-duality representations of the $(D-1)$-form and $D$-form potentials of maximal supergravity in $D<10$ dimensions have been determined by using the embedding tensor technique [6]. Independently, they have been determined [7, 8] by making use of the properties of the very extended Kac-Moody algebra $E_{11}$ [9]. The number of these potentials becomes quite large in lower dimensions.

In this paper we wish to address the question of which $p$-form potentials of maximal supergravities correspond to supersymmetric branes in (compactified) string theory. In doing so it is important to first specify which kind of branes we wish to consider. We will only consider branes for which we can construct a gauge-invariant Wess-Zumino (WZ) term describing the coupling of the supergravity potentials to the brane. The construction of such a gauge-invariant WZ term in itself is straightforward. All one needs is the transformation rules of the potentials under the different gauge symmetries where for every (pull-back of the) gauge parameters one introduces a corresponding worldvolume potential. We will impose the non-trivial requirement that these worldvolume potentials fit into a supermultiplet with 16 supercharges. The branes satisfying this condition have the necessary ingredients to make the construction of a kappa-symmetric worldvolume action possible. It is therefore suggestive that these branes are well-defined in string theory. ${ }^{2}$ We stress that this does not imply that the remaining $p$-form potentials have nothing to do with branes at all. Their mere existence as part of maximal supergravity is suggestive and perhaps, when a proper non-perturbative formulation of string theory has been developed, we will understand how these potentials couple to some class of branes.

It is insightful to classify the branes according to the way their tension $T$ scales with the string coupling constant $g_{s}$ in the string frame. This can be specified by an integer number $\alpha$ via

$$
T \sim\left(g_{s}\right)^{\alpha}
$$

It turns out that in string theory $\alpha \leq 0$. The highest values of $\alpha$ correspond to the following kind of branes:

$$
\alpha=0 \text { : Fundamental Branes; } \quad \alpha=-1 \text { : D-branes; } \quad \alpha=-2 \text { : Solitonic Branes . }
$$

Our understanding of the branes with $\alpha=-3,-4, \cdots$ is rather limited. In the lower dimensional theories, which result from dimensional reduction from eleven dimensions, we may write $g_{s}=<e^{\phi}>$ where the dilaton $\phi$ refers to any of the compactified directions. Selecting this dilaton direction corresponds to decomposing the $D$-dimensional U-duality group with respect to T-duality as

$$
\mathrm{E}_{11-D(11-D)} \supset \mathrm{SO}(10-D, 10-D) \times \mathbb{R}^{+} .
$$

\footnotetext{
${ }^{2}$ For branes with co-dimension 2 one needs to integrate over the moduli space to obtain finite-energy configurations. Branes with co-dimension 0, i.e. space-time filling branes, need to be combined with orientifold planes to define string theories with 16 supercharges. In this paper we will not consider these issues but only consider single branes by themselves.
} 
The dependence of the brane tension on this dilaton, i.e. the number $\alpha$, is determined by the rank $p$ of the corresponding $p$-form potential and the weight $w$ with which this potential transforms under the $\mathbb{R}^{+}$-symmetry [10]. ${ }^{3}$ This conserved number is thus defined for any supergravity field, regardless of whether it corresponds to a brane or not.

In this work we will consider the Wess-Zumino terms of branes in $D \leq 10$ dimensions for a fixed value of $\alpha$, i.e. according to T-duality representations. This is to be distinguished from the vast literature on S-duals of brane actions (including the kinetic terms) in $D=10$ dimensions, see, e.g., [13-18]. In our previous work we only considered the Fundamental Branes and D-branes of (compactified) string theory [10]. Since the Fundamental Branes have the highest value of $\alpha$, only Fundamental Branes themselves can end on them. In practice, we find that only Fundamental 0-Branes, i.e. wrapped strings, may end on the Fundamental String. The construction of a gauge-invariant WZ term therefore only requires world-volume scalars that fit into a scalar multiplet. In $D=10$ dimensions only embedding scalars are needed and the WZ term is given by the well-known expression

$$
\mathcal{L}_{\mathrm{WZ}}^{\mathrm{D}=10}(\text { Fundamental String })=B_{2},
$$

where $B_{2}$ is the (pull-back of the) NS-NS 2 -form. In $D<10$ wrapped strings can end on the Fundamental String and the corresponding WZ term gets accordingly modified with extra world-volume scalars [19-21]:

$$
\mathcal{L}_{\mathrm{WZ}}^{\mathrm{D}<10}(\text { Fundamental String })=B_{2}+\eta^{A B} \mathcal{F}_{1, A} B_{1, B}
$$

where $B_{1, A}$ are the NS-NS 1 -forms and $\mathcal{F}_{1, A}$ are the 1-form world-volume curvatures of the extra scalars. Both transform as a vector, indicated by the index $A$, under the Tduality group $\mathrm{SO}(d, d)$ with $d=10-D$. The number of extra scalars is twice the number of compactified dimensions in line with doubled geometry [19-21]. The WZ term for the Fundamental 0-branes themselves does not contain these extra scalars and is given by (omitting the explicit vector-index $A$ )

$$
\mathcal{L}_{\mathrm{WZ}}^{\mathrm{D}<10}(\text { Fundamental 0-Branes })=B_{1} .
$$

We next consider the D-branes. Since they have the one-to-highest value of $\alpha$, only Fundamental Branes can end on them. ${ }^{4}$ In $D=10$ dimensions there are only Fundamental Strings that can end on D-branes and, accordingly, the WZ term gets deformed by an extra Born-Infeld worldvolume vector, with 2 -form curvature $\mathcal{F}_{2}$ :

$$
\mathcal{L}_{\mathrm{WZ}}^{\mathrm{D}=10}(\mathrm{D} \text {-branes })=e^{\mathcal{F}_{2}} C .
$$

\footnotetext{
${ }^{3}$ The number $\alpha$ has a natural group theory interpretation in terms of the Kac-Moody algebra $\mathrm{E}_{11}$ [11] (see also [12]). We assume here that the brane tension contains a leading term which only depends on the dilaton. In general, this need not be the case. To determine the full dependence of $T$ on all fields we need the supersymmetry rules of the $p$-form potentials. We will not investigate this further in this work.

${ }^{4}$ Here and in the rest of the paper we only consider the objects that are electrically charged with respect to the worldvolume fields. For each such object there is a corresponding magnetic object that can end on the same brane. For instance, in the case of a $\mathrm{D} p$-brane, the magnetic version of the fundamental string is a $\mathrm{D}(p-2)$-brane and the magnetic version of a fundamental particle is a $\mathrm{D}(p-1)$-brane.
} 
Here $C$ stands for the formal sum of all RR potentials. In [10] we derived the T-dualitycovariant expression of the D-brane WZ terms in $D<10$ dimensions. Since now both wrapped and un-wrapped Fundamental Strings can end on the D-branes we get a further deformation by the extra worldvolume scalars [10]:

$$
\mathcal{L}_{\mathrm{WZ}}^{\mathrm{D}<10}(\mathrm{D} \text {-branes })=e^{\mathcal{F}_{2}} e^{\mathcal{F}_{1, A} \Gamma^{A}} C
$$

where $\Gamma^{A}$ are the gamma-matrices of $\mathrm{SO}(d, d)$. The reason for the existence of the general expression (1.8) is that in any dimension the fundamental potentials transform as a singlet (2-form) and vector (1-form) under T-duality while the D-brane potentials ( $p$-forms) transform as (chiral) spinor representations of the same duality group.

In this paper we wish to continue the analysis of [10] and consider the next set of branes, i.e. the String Solitons. The analysis becomes now more subtle due to two reasons. First of all, both Fundamental Branes as well as D-branes may end on String Solitons. This leads to many world-volume $p$-form potentials which must fit into one of the two available world-volume supermultiplets with 16 supercharges: the vector multiplet in ten dimensions or less (with 1 vector) or the six-dimensional tensor multiplet (with 1 selfdual 2-form potential). In general, we will obtain too many world-volume potentials to fit any of these two multiplets. There are two ways to lower the number of the world-volume potentials. One way is to impose world-volume duality relations. Another way is by making suitable redefinitions of the target space solitonic, i.e. $\alpha=-2$, potentials with terms that are quadratic in the $\alpha=-1 \mathrm{RR}$ potentials. In many cases this lowering of the number of world-volume potentials turns out not to be enough. Unlike the Fundamental Branes and the D-branes we find that many of the solitonic potentials of maximal supergravity do not satisfy our criterion that they must couple to a String Soliton via a gauge-invariant WZ term that only contains world-volume potentials that fit into a vector or a tensor multiplet. The fact that we find branes that satisfy our criterion is possible due to a beautiful and intricate interplay between target space and worldvolume supersymmetry and electromagnetic duality.

To classify the cases in which supersymmetric String Solitons exist we proceed in two steps. First, we determine the T-duality representations of the potentials that contain the ones that couple to supersymmetric String Solitons. We find that these are antisymmetric tensor representations. Next, we determine the conjugacy classes within the T-duality representations to which the supersymmetric String Solitons belong. We will show that these conjugacy classes are defined by a specific set of lightlike directions within the antisymmetric tensor representation. The phenomenon that branes only form a conjugacy class within a given T-duality representation does not occur for Fundamental Branes and D-branes simply because in these cases the T-duality representations involved do not contain non-trivial conjugacy classes. It has however a well-known analogue in the case of ten-dimensional 7-branes with respect to the S-duality group $\mathrm{SL}(2, \mathbb{R})$. The 8-form potentials of IIB supergravity that contain the potential that couples to the D7-brane are in the 3 representation of $\mathrm{SL}(2, \mathbb{R}){ }^{5}$ It turns out that the D7-brane and its S-dual belong to a

\footnotetext{
${ }^{5}$ The IIB supersymmetry algebra closes on a triplet of 8-forms provided that a duality relation with the
} 
2-dimensional conjugacy class within this triplet. The remaining component of the triplet does not correspond to a brane. To see this it is convenient to use a real $\mathrm{SO}(2,1)$ notation since that resembles most the discussion in the case of T-duality. The crucial point is that in the construction of the WZ term for the triplet of 7-branes there is a coupling between the curvatures of the worldvolume 1-forms and the target space 6-forms of the form

$$
\mathcal{L}_{\mathrm{WZ}}^{\mathrm{D}=10}(7 \text {-branes }) \sim A_{8, i}+\mathcal{F}_{2} \Gamma_{i} A_{6}+\cdots, \quad i=1,2,3 .
$$

Here $\mathcal{F}_{2}$ and $A_{6}$ transform as two-component spinors of $\mathrm{SO}(2,1)$, that contain the BornInfeld vector and the RR 6-form together with their S-duals, respectively. We want the $\mathcal{F}_{2} \Gamma_{i} A_{6}$ term to describe only a coupling between the Born-Infeld vector and the RR 6 -form or the S-dual version of this. In particular, worldvolume supersymmetry requires that the Born-Infeld vector and its S-dual do not occur at the same time. Therefore, we want the $2 \times 2$ gamma matrix $\Gamma_{i}$ to act as a projection operator which picks out the correct component of the 2-dimensional spinor $\mathcal{F}_{2}$. To achieve this it is convenient to introduce a lightcone basis $i=(+,-, 3)$. One can easily convince oneself that the lightlike directions $\Gamma_{+}$and $\Gamma_{-}$that square to zero define the conjugacy class containing the D7-brane and its S-dual, which is an $\alpha=-3$ object. We will confirm in this paper that the remaining solitonic 8-form potential $A_{8,3}$, which has weight $\alpha=-2$, does not couple to a supersymmetric String Soliton. ${ }^{6}$ Our analysis of the conjugacy classes with respect to T-duality is very similar to the S-duality discussion above except that one is now dealing with the group $\mathrm{SO}(d, d)$ instead of $\mathrm{SO}(2,1)$.

A second feature that makes the analysis of String Solitons more subtle is that, whereas all Fundamental Branes and D-branes in $D<10$ dimensions can be obtained via dimensional reduction of the $D=10$ Fundamental String and D-branes, the same is not the case for the String Solitons. One way to understand the 10-dimensional origin of all lower-dimensional T-duality multiplets of solitonic potentials is to add to the standard supergravity fields extra solitonic fields of mixed symmetry without upsetting the counting of degrees of freedom. At the end of this paper we will show that the ten-dimensional mixed-symmetry solitonic fields that are needed to generate all the solitonic fields in lower dimensions are precisely the ones predicted by the very extended Kac-Moody algebra $\mathrm{E}_{11}$. It is not understood how to realise the full IIA/IIB supersymmetry algebra on these mixed symmetry potentials. This can only be achieved for linearised supersymmetry. However, these mixed-symmetry fields contain important information in the sense that, after reduction, they give rise to standard $p$-form potentials on which the non-linear supersymmetry

two scalars of IIB supergravity holds [22, 23]. This relation implies that one combination of the curvatures of the three 8-form potentials (multiplied by scalar-dependent factors) vanishes, in agreement with the fact that the theory contains only two scalars. This relation does not play a role in the present discussion.

${ }^{6}$ Using $\operatorname{SL}(2, \mathbb{R})$ notation the conjugacy classes of $\operatorname{SL}(2, \mathbb{R})$ are labelled by $\operatorname{det} Q$ where $Q$ is the $2 \times 2$ charge matrix. The D7-brane and its $\mathrm{S}$-dual belong to the $\operatorname{det} Q=0$ conjugacy class while the remaining 8 -form potential, the one that does not correspond to a brane, belongs to a $\operatorname{det} Q<0$ conjugacy class, see the last reference of [13-18]. This is to be distinguished from the 7-branes at the three orbifold points in the $\operatorname{SL}(2, \mathbb{R})$ moduli space. One of these branes is the D7-brane and belongs to the $\operatorname{det} Q=0$ conjugacy class while the branes at the other 2 orbifold points belong to the $\operatorname{det} Q>0$ conjugacy class. The latter branes can be viewed as bound states of the D7-brane with the S-dual of the D7-brane or anti-D7 brane, see e.g. [24]. 
algebra can be realised. In this paper we will derive a useful procedure of how to collect all (i.e. both $p$-form and mixed-symmetry) fields, predicted by $\mathrm{E}_{11}$, for a given value of $\alpha$. We will find that for $\alpha$ odd the representations of the IIA and IIB fields differ but that for $\alpha$ even they coincide. This applies in particular to the fundamental and solitonic potentials.

It is instructive to see how in $D=10$ dimensions the construction of a gauge-invariant WZ term can be achieved with a supermultiplet of world-volume potentials. Both Type IIA and Type IIB string theory contain a single solitonic 6 -form potential $D_{6}$, which couples to the NS5A-brane and NS5B-brane, respectively. The corresponding gauge-invariant WZ terms are given by (see section 3 )

$$
\begin{aligned}
& \mathcal{L}_{\mathrm{WZ}}^{\mathrm{D}=10}(\text { NS5A-brane })=D_{6}+\mathcal{G}_{5} C_{1}-\mathcal{G}_{3} C_{3}+\mathcal{G}_{1} C_{5}, \\
& \mathcal{L}_{\mathrm{WZ}}^{\mathrm{D}=10}(\text { NS5B-brane })=D_{6}+\mathcal{G}_{4} C_{2}-\mathcal{G}_{2} C_{4},
\end{aligned}
$$

where $C_{p}$ is a target space $\mathrm{RR} p$-form potential and where we have introduced world-volume $p$-form potentials $c_{p}$ with curvatures $\mathcal{G}_{p+1}$. These expressions can be straightforwardly obtained by applying a Noether procedure, replacing each parameter in the transformation rule of $D_{6}$ by a corresponding world-volume potential. The NS5A-brane contains the worldvolume potentials $c_{0}, c_{2}$ and $c_{4}$. Imposing a duality relation between them we are left with a scalar and a self-dual 2-form. Together with the 4 embedding scalars these form the bosonic sector of an $8+8$ tensor multiplet in the six-dimensional worldvolume. The extra $c_{0}$ scalar indicates that this solitonic 5-brane has an 11-dimensional origin as the M5-brane. On the other hand, the NS5B-brane contains the worldvolume potentials $c_{1}$ and $c_{3}$. Imposing a duality relation between them we are left with one vector. Together with the 4 embedding scalars these form the bosonic sector of an $8+8$ vector multiplet in the six-dimensional worldvolume.

It turns out that in $D=10$ there are three more solitonic $p$-form potentials $D_{8}, D_{10}$ and $D_{10}^{\prime}$, see table 8 . However, unlike $D_{6}$, these potentials cannot be associated with a supersymmetric String Soliton. These findings are consistent with our earlier analysis of Supersymmetric String Solitons, see [2-4, 25] and the last reference of [13-18]. The criterion we imposed in these references was that a cancellation under supersymmetry was taking place between the Nambu-Goto kinetic term and the leading solitonic potential in the WZ term. This requirement leads to an expression for the brane tension and the BPS condition. The conclusion based upon this criterion was that no Supersymmetric Soliton could be associated with $D_{8}, D_{10}$ and $D_{10}^{\prime}$. In this work we reach the same conclusion based upon the independent criterion that a gauge-invariant WZ term must exist with worldvolume potentials that fit into a supersymmetry multiplet.

We find that in $D<10$ dimensions the above results generalise in a T-duality covariant way as follows. In $D$ dimensions the $T$-duality representations of the potentials of maximal supergravity that contain the potentials that couple to supersymmetric String Solitons are anti-symmetric tensor representations of the T-duality group. More precisely, we find that these potentials are $p$-forms, with $D-4 \leq p \leq 6$ if $D \geq 6$ and with $D-4 \leq p \leq D$ if $D<6$, transforming in the antisymmetric representation of rank $m=p+4-D \leq 4$ of the T-duality symmetry $\mathrm{SO}(10-D, 10-D)$. We have summarised this result in table 1 . As 


\begin{tabular}{|c|c|c|c|}
\hline$p$ & solitonic potential & vector/tensor & vector \\
\hline$D-4$ & $D_{D-4}$ & IIB/IIA & $4 \leq D<10$ \\
\hline$D-3$ & $D_{D-3, A}$ & $D=9$ & $3 \leq D<9$ \\
\hline$D-2$ & $D_{D-2, A B}$ & $D=8$ & $2 \leq D<8$ \\
\hline$D-1$ & $D_{D-1, A B C}$ & $D=7$ & $1 \leq D<7$ \\
\hline$D$ & $D_{D, A B C D}$ & $D=6$ & $0 \leq D<6$ \\
\hline
\end{tabular}

Table 1. The T-duality multiplets of solitonic potentials that contain the ones that couple to supersymmetric String Solitons. The indices $A, B, \ldots$ are vector indices of $\mathrm{SO}(d, d)$, and they are always meant to be antisymmetrised. The last two columns indicate for which dimensions the worldvolume dynamics is described by a vector/tensor multiplet or by vector multiplets only, see the text.

discussed above the Supersymmetric Solitons form conjugacy classes within these T-duality representations. These conjugacy classes are described in table 9. The 10-dimensional origin of these conjugacy classes comes from $D_{6}$, together with a number of mixed-symmetry fields. The fact that these conjugacy classes contain among its components reductions of the known supersymmetric NS5A and NS5B branes of string theory guarantees that all other components of the same conjugacy class correspond to Supersymmetric String Solitons as well. All the remaining T-duality multiplets that do not contain potentials that can be associated with supersymmetric String Solitons, see table 2, come from either $D_{8}, D_{10}$ and $D_{10}^{\prime}$, together with a number of the mixed symmetry fields, or from mixed symmetry fields only.

Only 5-brane String Solitons can have both vector and tensor world-volume multiplets. The corresponding 6-form potentials have the peculiarity of always transforming as the reducible representation of $\mathrm{SO}(d, d)$ with $d$ antisymmetric indices, which splits in self-dual and anti self-dual irreducible representations. We will see that the self-dual representations correspond to String Solitons with vector multiplets, like the NS5B-brane, whereas the anti-selfdual representations correspond to String Solitons with tensor multiplets, like the NS5A-brane:

$$
D_{6, A_{1} \cdots A_{d}}^{+}: \text {vector multiplets, } \quad D_{6, A_{1} \cdots A_{d}}^{-}: \text {tensor multiplets }
$$

The $D=10$ case is special in the sense that the two 6 -form potentials belong to two different theories (IIA and IIB), see table 1.

In all cases we find that the gauge-invariant WZ term of the Supersymmetric Solitons is given by (omitting the anti-symmetric $A$-indices)

$$
\mathcal{L}_{\mathrm{WZ}}^{\mathrm{D} \leq 10}(\text { susy soliton })=e^{\mathcal{F}_{1}}(D+\overline{\mathcal{G}} \Gamma C)
$$

where $C$ and $D$ represent the $\mathrm{RR}$ and solitonic target space potentials, respectively, while $\mathcal{F}_{1}$ and $\mathcal{G}$ are the worldvolume curvatures of the fundamental worldvolume scalars and RR worldvolume potentials, respectively. In this expression, whose precise form (containing all indices and signs) can be found in eq. (5.8), it is understood that the String Solitons form a conjugacy class within the full T-duality representation. This conjugacy class is defined by specifying a certain set of lightlike directions, see eq. (5.9). Formula (1.13), together 
with a description of the conjugacy classes, constitutes the main result of this paper. This formula includes the ten-dimensional expressions (1.10) and (1.11) as special cases with the understanding that in the IIA (IIB) case we only consider the even-form (odd-form) worldvolume potentials. The special feature of the WZ-term (1.13), which is only possible for the antisymmetric T-duality representations of table 1 , is that the gauge-invariance of the WZ-term can be achieved without introducing a Born-Infeld vector $b_{1}$ on the world-volume, i.e. no fundamental string can end on these supersymmetric String Solitons. For all the other T-duality multiplets, see table 2, one must introduce this Born-Infeld vector and this leads to too many worldvolume potentials that cannot fit into a vector or tensor multiplet.

This paper is organised as follows. In section 2 we derive in any dimension the solitonic potentials by decomposing the U-duality representations of all the potentials of any maximal supergravity theory with respect to T-duality. The result is summarised in table 2 . In section 3 we review and discuss the properties of the ten-dimensional String Solitons. In particular, we show why the solitonic potentials $D_{8}, D_{10}$ and $D_{10}^{\prime}$ do not couple to supersymmetric String Solitons. Next, in section 4, we derive the gauge transformations of all solitonic potentials in $D<10$ dimensions. This will be used in section 5 to derive the different gauge-invariant WZ terms of all $D<10$ String Solitons. The final result is given in eq. (5.8). In the same section we define the lightlike directions that define the conjugacy class to which the supersymmetric String Solitons belong, see eq. (5.9). In section 6 we show that our results agree with the classification of central charges of the supersymmetry algebra in any dimension. In section 7 we discuss how a possible ten-dimensional origin of the $D<10$ solitons leads one to consider mixed-symmetry fields in ten dimensions hinting at an underlying $E_{11}$ structure. We explain a procedure of how to extract out of $E_{11}$ all fields of IIA and IIB, both $p$-forms and mixed symmetry fields, for a given value of $\alpha$. As a side-result we will show that for IIA/IIB these fields coincide for even $\alpha$ but are different for odd $\alpha$. Finally, in section 8 we present our conclusions and discuss different consequences of our work. We also include an appendix, in which we discuss the properties of the spinors of $\mathrm{SO}(d, d)$ that are used in sections 4 and 5 .

\section{Solitonic fields in $D<10$ dimensions}

In recent years it has been established that maximal supergravity theories do not only contain $p$-form potentials, together with their duals, that describe physical degrees of freedom but also $(D-1)$-form and $D$-form potentials that do not describe any physical degree of freedom. The U-duality representations of these $p$-form potentials have been established by different means, all leading to the same answer. In $D=10$ dimensions all 9 -form and 10-form potentials that are consistent with the closure of the IIA or IIB supersymmetry algebra have been determined [2-4]. Upon toroidal reduction this leads to similar $(D-1)$ form and $D$-form potentials in $D<10$ dimensions. Their U-duality representations have been independently determined by a classification of gauged maximal supergravities [6] as well as by making use of $\mathrm{E}_{11}$ techniques $[7,8]$.

In this section we will derive the T-duality representations of the solitonic potentials in any dimensions by decomposing these U-duality representations with respect to T-duality 


\begin{tabular}{|c|c|}
\hline$(D-4)$-form & $D_{D-4}$ \\
\hline$(D-3)$-form & $D_{D-3, A}$ \\
\hline$(D-2)$-form & $D_{D-2}+D_{D-2, A B}$ \\
\hline$(D-1)$-form & $D_{D-1, A}+D_{D-1, A B C}$ \\
\hline$D$-form & $D_{D}+D_{D}^{\prime}+D_{D, A B}+D_{D, A B C D}$ \\
\hline
\end{tabular}

Table 2. Forms with $\alpha=-2$ in any dimension $D \geq 3$ (In three dimensions the first line is absent because the corresponding field does not exist). The indices $A, B, \ldots$ are vector indices of $\mathrm{SO}(d, d)$, and they are always meant to be antisymmetrised.

according to eq. (1.3) and by picking out the solitonic fields. In any dimension $\alpha$, the number that determines how the brane tension scales with the string coupling constant, is related to the $\mathbb{R}^{+}$weight in the decomposition (1.3) and to the rank of the form. We will explicitly perform our analysis in dimensions higher than four, but as we will see in section 7 our results are general and apply to four and three dimensions as well. The resulting T-duality representations take on a universal form for any dimension which we have summarised in table 2. Here and in the rest of the paper we denote the solitonic fields with $D$, simply because the $\alpha=0$ fields and the $\alpha=-1$ fields are denoted in the literature and in this paper with $B$ and $C$ respectively. The solitonic fields should not be confused with the dimension of spacetime, which is also denoted with $D$. Actually, in [10] the decomposition of the U-duality representations with respect to the T-duality group $\mathrm{SO}(d, d)$ was already performed, and the D-brane fields with $\alpha=-1$ were listed. Here we have refined that analysis. We have listed the result in tables 3-7 where we have given the value of $\alpha$ corresponding to all T-duality representations in dimensions $5 \leq D \leq 9$. In these tables $w$ denotes the $\mathbb{R}^{+}$weight, and the U-duality group decomposes as in eq. (1.3). The conventions for the representations and the $w$ weights are those of [10], which, in turn, are taken from [26].

We now proceed with an analysis of all the $\alpha=-2$ fields listed in tables 3-7 and summarized in table 2 . In all cases, the form of lowest rank is a $D-4$ form, that transforms as a singlet under T-duality. This form is the magnetic dual of the Fundamental 2-form $B_{2}$, and we denote it by $D_{D-4}$.

Increasing the rank by one unit, one can see from the tables that in any dimension one has solitonic $D-3$-forms transforming as vectors under T-duality. These fields are the duals of the Fundamental fields $B_{1, A}$, and we denote them by $D_{D-3, A}$. A particular case is the nine-dimensional one, in which the T-duality group is $\operatorname{SO}(1,1)$ and the vector representation splits into its selfdual and anti-selfdual part. This can be seen in table 3 , which shows that the U-duality representation of the 6 -form is reducible and each of the two irreducible components contain either the selfdual or the anti-selfdual representation when decomposed under T-duality.

The T-duality representation of the solitonic $D-2$-forms is reducible in all cases. The reader can derive in all cases by looking at the tables that the fields are $D_{D-2}$ and $D_{D-2, A B}$, transforming as a singlet and as an antisymmetric tensor respectively under Tduality. In $D=9$ these are actually two singlets, and each singlet arises from each of the 


\begin{tabular}{|c|c|c|c|c|c|c|}
\hline field & $\mathrm{U}$ repr & $\alpha=0$ & $\alpha=-1$ & $\alpha=-2$ & $\alpha=-3$ & $\alpha=-4$ \\
\hline \multirow{2}{*}{ 1-form } & $\mathbf{2}_{0}$ & $(1,0)$ & $(-1,0)$ & & & \\
\cline { 2 - 7 } & $\mathbf{1}_{1}$ & $(0,1)$ & & & & \\
\hline 2-form & $\mathbf{2}_{1}$ & $(1,1)$ & $(-1,1)$ & & & \\
\hline 3-form & $\mathbf{1}_{1}$ & & $(0,1)$ & & & \\
\hline 4-form & $\mathbf{1}_{2}$ & & $(0,2)$ & & & \\
\hline 5-form & $\mathbf{2}_{2}$ & & $(1,2)$ & $(-1,2)$ & & \\
\hline 6-form & $\mathbf{2}_{3}$ & & $(1,3)$ & $(-1,3)$ & & \\
\cline { 2 - 7 } & $\mathbf{1}_{2}$ & & & $(0,2)$ & & \\
\hline 7-form & $\mathbf{3}_{3}$ & & $(2,3)$ & $(0,3)$ & $(-2,3)$ & \\
\cline { 2 - 7 } & $\mathbf{1}_{3}$ & & & $(0,3)$ & & \\
\hline 8-form & $\mathbf{3}_{4}$ & & $(2,4)$ & $(0,4)$ & $(-2,4)$ & \\
\cline { 2 - 7 } & $\mathbf{2}_{3}$ & & & $(1,3)$ & $(-1,3)$ & \\
\hline 9-form & $\mathbf{4}_{4}$ & & $(3,4)$ & $(1,4)$ & $(-1,4)$ & $(-3,4)$ \\
\cline { 2 - 7 } & $2 \times \mathbf{2}_{4}$ & & & $2 \times(1,4)$ & $2 \times(-1,4)$ & \\
\hline
\end{tabular}

Table 3. The decomposition of the $n$-form potentials of $D=9$ maximal supergravity. The Uduality is $\operatorname{SL}(2, \mathbb{R}) \times \mathbb{R}^{+}$. We denote with $\left(w_{1}, w_{2}\right)$ the weights associated to $\mathbb{R}^{+} \times \mathbb{R}^{+}$. The weight under T-duality is $w_{1}-w_{2}$. The value of $\alpha$ is given by $\alpha=\frac{1}{2}\left(w_{1}+w_{2}-n\right)$.

two irreducible U-duality representations of the 7 -forms. In $D=8$ the singlet and the selfdual part of $D_{6, A B}$ arise from the decomposition of the 6 -form in the $(\mathbf{8}, \mathbf{1}) \mathrm{U}$-duality representation, while the anti-selfdual part of $D_{6, A B}$ is the 6-form in the $(\mathbf{1}, \mathbf{3}) \mathrm{U}$-duality representation. In dimensions lower than eight $D_{D-2}$ and $D_{D-2, A B}$ arise from the same U-duality representation.

Proceeding in the same way, one finds that the solitonic $D-1$-forms are $D_{D-1, A}$ and $D_{D-1, A B C}$, that is a vector and an antisymmetric tensor with three indices under Tduality. In seven dimensions the field $D_{D-1, A B C}$ splits into a selfdual and an anti-selfdual part. Clearly, such a three-index antisymmetric tensor does not occur in nine dimensions due to the fact that the corresponding $\mathrm{SO}(1,1)$ T-duality representation does not exist.

Finally, one can see that in all cases the solitonic $D$-forms are two singlets $D_{D}$ and $D_{D}^{\prime}$, an antisymmetric tensor with two indices $D_{D, A B}$ and and antisymmetric tensor with four indices $D_{D, A B C D}$. The latter field does not exist in nine dimensions. To summarise, one can see from the tables that the column corresponding to $\alpha=-2$ gives in all cases the general result given in table 2 .

There are some general conclusions that one can draw by looking at the representations occurring in table 2. First of all, the 6-forms have the peculiarity of always containing the reducible representation of $\mathrm{SO}(d, d)$ with $d$ antisymmetric indices, which splits in self-dual and anti self-dual irreducible representations. As we will see, this corresponds to the fact that the world-volume multiplet for a 5-brane can either be a vector or a tensor multiplet. The representations of the forms of rank higher than 6 , which can only occur in 7,8 and 9 dimensions, always occur at least in pairs. In nine dimensions the T-duality group is $\mathrm{SO}(1,1)$, which means that out of the fields in table 2 , only those with at most 2 indices 


\begin{tabular}{|c|c|c|c|c|c|c|}
\hline field & $\mathrm{U}$ repr & $\alpha=0$ & $\alpha=-1$ & $\alpha=-2$ & $\alpha=-3$ & $\alpha=-4$ \\
\hline 1-form & $(\overline{\mathbf{3}}, \mathbf{2})$ & $(\mathbf{2}, \mathbf{2})_{1}$ & $(\mathbf{1}, \mathbf{2})_{-2}$ & & & \\
\hline 2-form & $(\mathbf{3}, \mathbf{1})$ & $(\mathbf{1}, \mathbf{1})_{2}$ & $(\mathbf{2}, \mathbf{1})_{-1}$ & & & \\
\hline 3-form & $(\mathbf{1}, \mathbf{2})$ & & $(\mathbf{1}, \mathbf{2})_{0}$ & & & \\
\hline 4-form & $(\overline{\mathbf{3}}, \mathbf{1})$ & & $(\mathbf{2}, \mathbf{1})_{1}$ & $(\mathbf{1}, \mathbf{1})_{-2}$ & & \\
\hline 5-form & $(\mathbf{3}, \mathbf{2})$ & & $(\mathbf{1}, \mathbf{2})_{2}$ & $(\mathbf{2}, \mathbf{2})_{-1}$ & & \\
\hline 6-form & $(\mathbf{8}, \mathbf{1})$ & & $(\mathbf{2}, \mathbf{1})_{3}$ & $(\mathbf{1}, \mathbf{1})_{0}+(\mathbf{3}, \mathbf{1})_{0}$ & $(\mathbf{2}, \mathbf{1})_{-3}$ & \\
\cline { 2 - 8 } & $(\mathbf{1}, \mathbf{3})$ & & & $(\mathbf{1}, \mathbf{3})_{0}$ & & \\
\hline 7-form & $(\mathbf{6}, \mathbf{2})$ & & $(\mathbf{1}, \mathbf{2})_{4}$ & $(\mathbf{2}, \mathbf{2})_{1}$ & $(\mathbf{3}, \mathbf{2})_{-2}$ & \\
\cline { 2 - 7 } & $(\overline{\mathbf{3}}, \mathbf{2})$ & & & $(\mathbf{2}, \mathbf{2})_{1}$ & $(\mathbf{1}, \mathbf{2})_{-2}$ & \\
\hline \multirow{3}{*}{ 8-form } & $(\mathbf{1 5}, \mathbf{1})$ & & $(\mathbf{2}, \mathbf{1})_{5}$ & $(\mathbf{3}, \mathbf{1})_{2}+(\mathbf{1}, \mathbf{1})_{2}$ & $(\mathbf{4}, \mathbf{1})_{-1}+(\mathbf{2}, \mathbf{1})_{-1}$ & $(\mathbf{3}, \mathbf{1})_{-4}$ \\
\cline { 2 - 7 } & $(\mathbf{3}, \mathbf{3})$ & & & $(\mathbf{1}, \mathbf{3})_{2}$ & $(\mathbf{2}, \mathbf{3})_{-1}$ & \\
\cline { 2 - 7 } & $2 \times(\mathbf{3}, \mathbf{1})$ & & & $2 \times(\mathbf{1}, \mathbf{1})_{2}$ & $2 \times(\mathbf{2}, \mathbf{1})_{-1}$ & \\
\hline
\end{tabular}

Table 4. The decomposition of the $n$-form potentials of $D=8$ maximal supergravity. The Uduality symmetry is $\operatorname{SL}(3, \mathbb{R}) \times \mathrm{SL}(2, \mathbb{R})$ and the T-duality is $\mathrm{SL}(2, \mathbb{R}) \times \mathrm{SL}(2, \mathbb{R})$, while the subscript denotes the $\mathbb{R}^{+}$- charge $w$, which is related to $\alpha$ by the equation $\alpha=-\frac{1}{3}(n-w)$.

\begin{tabular}{|c|c|c|c|c|c|c|}
\hline field & $\mathrm{U}$ repr & $\alpha=0$ & $\alpha=-1$ & $\alpha=-2$ & $\alpha=-3$ & $\alpha=-4$ \\
\hline 1-form & $\overline{\mathbf{1 0}}$ & $\mathbf{6}_{2}$ & $\overline{\mathbf{4}}_{-3}$ & & & \\
\hline 2-form & $\mathbf{5}$ & $\mathbf{1}_{4}$ & $\mathbf{4}_{-1}$ & & & \\
\hline 3-form & $\overline{\mathbf{5}}$ & & $\overline{\mathbf{4}}_{1}$ & $\mathbf{1}_{-4}$ & & \\
\hline 4-form & $\mathbf{1 0}$ & & $\mathbf{4}_{3}$ & $\mathbf{6}_{-2}$ & & \\
\hline 5-form & $\mathbf{2 4}$ & & $\overline{\mathbf{4}}_{5}$ & $\mathbf{1 5}_{0}+\mathbf{1}_{0}$ & $\mathbf{4}_{-5}$ & \\
\hline 6-form & $\overline{\mathbf{4 0}}$ & & $\mathbf{4}_{7}$ & $\mathbf{1 0}_{2}+\mathbf{6}_{2}$ & $\overline{\mathbf{2 0}}_{-3}$ & \\
\cline { 2 - 7 } & $\overline{\mathbf{1 5}}$ & & & $\overline{\mathbf{1 0}}_{2}$ & $\overline{\mathbf{4}}_{-3}$ & $\mathbf{1}_{-8}$ \\
\hline 7-form & $\mathbf{7 0}$ & & $\overline{\mathbf{4}}_{9}$ & $\mathbf{1 5}_{4}+\mathbf{1}_{4}$ & $\mathbf{3 6}_{-1}+\mathbf{4}_{-1}$ & $\mathbf{1 0}_{-6}$ \\
\cline { 2 - 7 } & $\mathbf{4 5}$ & & & $\mathbf{1 5}_{4}$ & $\mathbf{2 0}_{-1}+\mathbf{4}_{-1}$ & $\mathbf{6}_{-6}$ \\
\cline { 2 - 7 } & $\mathbf{5}$ & & & $\mathbf{1}_{4}$ & $\mathbf{4}_{-1}$ & \\
\hline
\end{tabular}

Table 5. The decomposition of the $n$-form potentials of $D=7$ maximal supergravity. The Uduality group is $\operatorname{SL}(5, \mathbb{R})$ and the T-duality group is $\operatorname{SL}(4, \mathbb{R})$. We denote as a subscript the $\mathbb{R}^{+}$ charge (notation from [26]). The value of $\alpha$ is given by $\alpha=\frac{1}{5}(w-2 n)$.

survive. The representations of the 7 -forms are two singlets, the representation of the 8 -form is a vector which decomposes in self-dual and anti self-dual representations, and finally the 9-forms are three singlets. In eight dimensions the T-duality is $\mathrm{SO}(2,2)$, which means that the two representations of the 7 -forms are the same, while the representation with four indices of the 8-form is a scalar and the representation with two indices splits in self-dual and anti self-dual. In seven dimensions the T-duality is $\mathrm{SO}(3,3)$, and thus the representation of the 7 -form with two indices and the one with four indices coincide. 


\begin{tabular}{|c|c|c|c|c|c|c|c|}
\hline field & U repr & $\alpha=0$ & $\alpha=-1$ & $\alpha=-2$ & $\alpha=-3$ & $\alpha=-4$ & $\alpha=-5$ \\
\hline 1-form & 16 & $\left(8_{\mathrm{C}}\right)_{1}$ & $\left(\mathbf{8}_{\mathrm{S}}\right)_{-1}$ & & & & \\
\hline 2 -form & 10 & $\mathbf{1}_{2}$ & $\left(\mathbf{8}_{\mathrm{V}}\right)_{0}$ & $\overline{\mathbf{1}_{-2}}$ & & & \\
\hline 3 -form & $\overline{\mathbf{1 6}}$ & & $\left(8_{\mathrm{S}}\right)_{1}$ & $\left(8_{\mathrm{C}}\right)_{-1}$ & & & \\
\hline 4-form & 45 & & $\left(8_{\mathrm{V}}\right)_{2}$ & $28_{0}+\mathbf{1}_{0}$ & $\left(8_{\mathrm{V}}\right)_{-2}$ & & \\
\hline 5 -form & 144 & & $\left(8_{\mathrm{S}}\right)_{3}$ & $\left(\mathbf{8}_{\mathrm{C}}\right)_{1}+\left(\mathbf{5 6}_{\mathrm{C}}\right)_{1}$ & $\left(\mathbf{8}_{\mathrm{S}}\right)_{-1}+\left(\mathbf{5} \mathbf{6}_{\mathrm{S}}\right)_{-1}$ & $\left(\mathbf{8}_{\mathrm{C}}\right)_{-3}$ & \\
\hline \multirow[t]{3}{*}{ 6-form } & 320 & & $\left(\mathbf{8}_{\mathrm{V}}\right)_{4}$ & $\begin{array}{c}\left(\mathbf{3 5} \mathbf{V}_{\mathrm{V}}\right)_{2}+\mathbf{2 8} \mathbf{8}_{2} \\
+\mathbf{1}_{2}\end{array}$ & $\begin{array}{l}2 \times\left(\mathbf{8}_{\mathrm{V}}\right)_{0} \\
+\left(\mathbf{1 6 0} \mathbf{V}_{\mathrm{V}}\right)_{0}\end{array}$ & $\begin{array}{c}\left(\mathbf{3 5}_{\mathrm{V}}\right)_{-2}+ \\
\mathbf{2 8}_{-2}+\mathbf{1}_{-2}\end{array}$ & $\left(8_{V}\right)_{-4}$ \\
\hline & $\overline{\overline{126}}$ & & & $\left(35_{\mathrm{S}}\right)_{2}$ & $\left(5 \mathbf{6}_{\mathrm{V}}\right)_{0}$ & $\left(35_{C}\right)_{-2}$ & \\
\hline & 10 & & & $\mathbf{1}_{2}$ & $\left(\mathbf{8}_{\mathrm{V}}\right)_{0}$ & $\overline{\mathbf{1}_{-2}}$ & \\
\hline
\end{tabular}

Table 6. The decomposition of the $n$-form potentials of $D=6$ maximal supergravity. The Uduality is $\mathrm{SO}(5,5)$, while the T-duality is $\mathrm{SO}(4,4)$. The relation between $\alpha$ and $w$ is $\alpha=\frac{1}{2}(w-n)$.

\begin{tabular}{|c|c|c|c|c|c|c|c|}
\hline field & $\mathrm{U}$ repr & $\alpha=0$ & $\alpha=-1$ & $\alpha=-2$ & $\alpha=-3$ & $\alpha=-4$ & $\alpha=-5$ \\
\hline 1-form & $\mathbf{2 7}$ & $\mathbf{1 0}_{-2}$ & $\mathbf{1 6}_{1}$ & $\mathbf{1}_{4}$ & & & \\
\hline 2-form & $\overline{\mathbf{2 7}}$ & $\mathbf{1}_{-4}$ & $\overline{\mathbf{1 6}}_{-1}$ & $\mathbf{1 0}_{2}$ & & & \\
\hline 3-form & $\mathbf{7 8}$ & & $\mathbf{1 6}_{-3}$ & $\mathbf{4 5}_{0}+\mathbf{1}_{0}$ & $\overline{\mathbf{1 6}}_{3}$ & & \\
\hline 4-form & $\mathbf{3 5 1}$ & & $\overline{\mathbf{1 6}}_{-5}$ & $\mathbf{1 2 0}_{-2}+\mathbf{1 0}_{-2}$ & $\mathbf{1 6}_{1}+\mathbf{1 4 4}_{1}$ & $\mathbf{4 5}_{4}$ & \\
\hline 5-form & $\overline{\mathbf{1 7 2 8}}$ & & $\mathbf{1 6}_{-7}$ & $\begin{array}{c}\mathbf{1}_{-4}+\mathbf{4 5}_{-4} \\
+\mathbf{2 1 0}_{-4}\end{array}$ & $\overline{\mathbf{1 4 4}}_{-1}+\overline{\mathbf{1 6}}_{-1}$ & $\mathbf{1 0}_{2}+\mathbf{1 2 0}_{2}+$ & $\mathbf{1 4 4}_{5}$ \\
\cline { 2 - 8 } & $\overline{\mathbf{2 7}}$ & & & $\mathbf{1}_{-4}+\overline{\mathbf{1 2 6}}_{2}$ & \\
\hline
\end{tabular}

Table 7. The decomposition of the $n$-form potentials of $D=5$ maximal supergravity. The Uduality is $\mathrm{E}_{6}$ and the T-duality is $\mathrm{SO}(5,5)$. The relation between $\alpha$ and the weight $w$ is given by the equation $\alpha=-\frac{1}{3}(w+2 n)$.

\section{String solitons in ten dimensions}

The purpose of this section is to consider the solitonic $D$-fields of IIA and IIB supergravity and to investigate which of these fields can couple to a solitonic brane with a worldvolume supersymmetric field content. We have summarised these $D$-fields in table 8 . The results of this investigation will not be surprising: we will find that the only solitonic branes are the NS5A-brane, with a worldvolume tensor multiplet, and the NS5B-brane, with a worldvolume vector multiplet. More precisely, only one of the 4 solitonic IIA (IIB) $D$-fields in table 8 corresponds to a supersymmetric solitonic IIA (IIB) brane.

An important lesson we learn from this analysis is that, even in ten dimensions, the requirement of a worldvolume supersymmetric field content excludes most of the $D$-fields as potentials suggesting new solitonic branes. The status of these $D$-fields at present is unclear. As already stressed in the introduction this does not necessarily imply that these $D$-fields have nothing to do with branes at all. In this paper, however, we will restrict our attention to the solitonic branes of string theory that have a known supersymmetric worldvolume field content. 


\begin{tabular}{|c|c|c|}
\hline field & IIA brane/multiplet & IIB brane/multiplet \\
\hline$D_{6}$ & NS5A/tensor multiplet & NS5B/vector multiplet \\
\hline$D_{8}$ & - & - \\
\hline$D_{10}, D_{10}^{\prime}$ & - & - \\
\hline
\end{tabular}

Table 8. The solitonic $D$-fields of IIA and IIB supergravity. The second and third column indicate which $D$-fields couple to the known solitonic branes of string theory. The worldvolume multiplets of these branes are indicated.

The restriction we find on the allowed solitonic $D$-fields results from a clash between the known supersymmetric worldvolume multiplets with 16 supercharges, i.e. the vector multiplet and the tensor multiplet, and the number of worldvolume fields that we need to construct a gauge-invariant Wess-Zumino term. Since the tensor multiplet only exists in 6 dimensions, only 5-branes can have a worldvolume dynamics governed by a tensor multiplet. Both fundamental objects as well as D-branes can end on the Solitonic Brane and this leads to many worldvolume $p$-form potentials. It is non-trivial to fit all these potentials into a vector or tensor multiplet. Since vector multiplets already occur in the case of D-branes we expect these solitonic branes to be the S-duals of D-branes. In contrast, we expect the solitonic 5-branes with tensor multiplets to be related, via dimensional reduction, to the M5-brane of M-theory which also has a worldvolume tensor multiplet.

We now consider the occurrence of worldvolume $p$-form fields for the ten-dimensional solitonic branes in more detail and show in which cases they fit a vector or tensor multiplet. Both IIA and IIB supergravity contain the Fundamental 2-form potential $B_{2}$, with curvature $H_{3}$, that couples to the Fundamental String. They differ however in the RR-potentials in the sense that IIA (IIB) supergravity contain odd-form (even-form) RR potentials. All RR potentials couple to the D-branes. Using the same compact notation as in [10] we can write the transformation rules of the IIA and IIB RR potentials and the expressions for their curvatures as

$$
\delta C=d \lambda+H_{3} \lambda
$$

and

$$
G=d C+H_{3} C,
$$

respectively. Here $\lambda$ denote the $\mathrm{RR}$ gauge parameters. It is understood that in the IIA case we pick out the odd-form potentials whereas in the IIB case we take the even-form potentials. One then introduces a worldvolume 1-form field $b_{1}$ associated to the fundamental 2 -form $B_{2}$, whose gauge-invariant curvature is

$$
\mathcal{F}_{2}=d b_{1}+B_{2},
$$

and constructs a gauge-invariant WZ-term which was written in the introduction in eq. (1.7).

Using the same short-hand notation the gauge transformations of the solitonic $D$-fields can be written as (here $G \lambda$ denotes the various contributions of the right rank; we will give precise coefficient of this term in the two subsections below)

$$
\delta D=d \Lambda+G \lambda+H_{3} \Lambda,
$$


where $\Lambda$ denote the solitonic gauge parameters. The corresponding gauge-invariant curvatures are given by

$$
F=d D+G C+H_{3} D
$$

where again $G C$ schematically denotes the various terms such that the rank of $G$ plus the rank of $C$ equals the rank of $F$.

Given that the RR field strengths occur in the gauge transformations of the $D$ fields, in order to write down a gauge-invariant WZ term one introduces worldvolume fields $c$ associated to the RR fields. These worldvolume fields are even forms for IIA and odd-forms for IIB. Note that this is the opposite of the target-space potentials. The gauge-invariant worldvolume curvatures for the $c$ potentials are given by

$$
\mathcal{G}=d c+H_{3} c+C,
$$

with $\delta c=-\lambda$. They satisfy the Bianchi identities

$$
d \mathcal{G}=-H_{3} \mathcal{G}+G .
$$

Using the above formulae one can show that the following candidate WZ-term is (trivially) gauge-invariant:

$$
\mathcal{L}_{\mathrm{WZ}}(\text { solitonic })=e^{\mathcal{F}_{2}}(D+\mathcal{G} C) .
$$

The danger of this Ansatz is that it contains many worldvolume potentials hidden in the worldvolume curvatures $\mathcal{F}_{2}$ and $\mathcal{G}$ and they are difficult to fit into a vector or tensor multiplet.

Below we will scan the different cases for IIA and IIB supergravity separately, give the precise formulae, and see in which cases we can put all worldvolume fields into a given multiplet.

\subsection{The IIB solitonic WZ terms}

We first consider the 6 -form potential $D_{6}$ corresponding to the NS5B-brane. The gauge transformations and curvatures are given by

$$
\delta D_{6}=d \Lambda_{5}+G_{5} \lambda_{1}-G_{3} \lambda_{3}
$$

and

$$
F_{7}=d D_{6}+G_{5} C_{2}-G_{3} C_{4},
$$

respectively. The gauge-invariant $\mathrm{WZ}$ term is given by

$$
\mathcal{L}_{\mathrm{WZ}}=D_{6}+\mathcal{G}_{4} C_{2}-\mathcal{G}_{2} C_{4} \equiv \mathcal{D}_{6},
$$

which contains the worldvolume potentials $c_{1}$ and $c_{3}$. Imposing a duality relation between them we are left with one vector. Together with the 4 embedding scalars these form the 8 bosonic degrees of freedom of an $8+8$ vector multiplet in the six-dimensional worldvolume.

We next consider the solitonic 8-form $D_{8}$. An important difference with the previous case is that $D_{8}$ transforms to $H_{3}$ under a solitonic gauge transformation. Basically, 
this means that fundamental strings can end on this soliton and, hence, there exists a corresponding Born-Infeld vector $b_{1}$ in the worldvolume. As we will see, this will lead to too many worldvolume fields to fit a multiplet. Partially, this effect is cancelled (but not enough) in this case due to the fact that the transformation rule and curvature contain a free real parameter $\alpha$. Indeed, the general form of the gauge transformation and field strength in eqs. (3.4) and (3.5), requiring gauge invariance of the field strength leads to

$$
\delta D_{8}=d \Lambda_{7}+(1-\alpha) G_{7} \lambda_{1}+\alpha G_{5} \lambda_{3}-(1+\alpha) G_{3} \lambda_{5}+H_{3} \Lambda_{5}
$$

and

$$
F_{9}=d D_{8}+(1-\alpha) G_{7} C_{2}+\alpha G_{5} C_{4}-(1+\alpha) G_{3} C_{6}+H_{3} D_{6} .
$$

The free parameter $\alpha$ can be reabsorbed by the redefinition

$$
D_{8} \rightarrow D_{8}+\alpha\left(C_{2} C_{6}-\frac{1}{2} C_{4} C_{4}\right) .
$$

That this is an allowed redefinition can be seen from the fact that

$$
d\left(C_{2} C_{6}-\frac{1}{2} C_{4} C_{4}\right)=G_{3} C_{6}+G_{7} C_{2}-G_{5} C_{4},
$$

which means that the terms $H_{3} C C$ vanish, and therefore adding this term preserves the structure of eq. (3.5). A gauge-invariant WZ term for this case is given by

$$
\mathcal{L}_{\mathrm{WZ}}=D_{8}+(1-\alpha) \mathcal{G}_{6} C_{2}+\alpha \mathcal{G}_{4} C_{4}-(1+\alpha) \mathcal{G}_{2} C_{6}+\mathcal{F}_{2} \mathcal{D}_{6} \equiv \mathcal{D}_{8}+\mathcal{F}_{2} \mathcal{D}_{6}
$$

which contains the worldvolume fields $c_{1}, c_{3}, c_{5}$ and $b_{1}$. The problem is now that there are too many worldvolume fields to fit into a multiplet. Even if we impose a duality relation between $c_{1}$ and $c_{5}$ we are left with two vectors and one 3 -form potential. The free parameter $\alpha$ may be used to eliminate one of the vectors or the 3 -form potential but not both. Hence, for no choice of $\alpha$ will the worldvolume fields fit into a vector multiplet. We can without loss of generality choose a value for the parameter $\alpha$, and we choose it to vanish, so that the field strength and gauge transformation are symmetric with respect to electromagnetic duality on the worldvolume, that is

$$
\delta D_{8}=d \Lambda_{7}+G_{7} \lambda_{1}-G_{3} \lambda_{5}+H_{3} \Lambda_{5} .
$$

Finally, we consider the 10 -form potentials. These are determined by formally requiring that their field strength is gauge invariant, although this is actually identically zero. Taking $\alpha=0$ in eq. (3.12), that is using eq. (3.17), one obtains

$$
\delta D_{10}=d \Lambda_{9}+\beta G_{9} \lambda_{1}+(1-\beta) G_{7} \lambda_{3}-(1-\beta) G_{5} \lambda_{5}-\beta G_{3} \lambda_{7}+H_{3} \Lambda_{7},
$$

where $\beta$ is an arbitrary real parameter. Given that no field redefinition is possible in this case, this parameter is the relative normalisation of the two independent 10 -forms. The corresponding gauge-invariant WZ term is given by

$$
\mathcal{L}_{\mathrm{WZ}}=D_{10}+\beta \mathcal{G}_{8} C_{2}+(1-\beta) \mathcal{G}_{6} C_{4}-(1-\beta) \mathcal{G}_{4} C_{6}-\beta \mathcal{G}_{2} C_{8}+\mathcal{F}_{2} \mathcal{D}_{8}+\frac{1}{2} \mathcal{F}_{2} \mathcal{F}_{2} \mathcal{D}_{6}
$$

This WZ term contains the potentials $c_{1}, c_{3}, c_{5}, c_{7}$ and $b_{1}$, which after imposing duality relations on the $c$ fields corresponds to a vector (multiplied by $\beta$ ) and a 3 -form (multiplied by $1-\beta$ ) plus another scalar. For no choice of the parameter $\beta$ can the field content be the bosonic sector of a ten-dimensional vector multiplet. 


\subsection{The IIA solitonic WZ terms}

We next consider the solitonic potentials of IIA supergravity (for zero Romans mass parameter). We first consider the 6 -form potential $D_{6}$ corresponding to the NS5A brane. The gauge transformations and curvature for this case are given by

$$
\delta D_{6}=d \Lambda_{5}+G_{6} \lambda_{0}-G_{4} \lambda_{2}+G_{2} \lambda_{4}
$$

and

$$
F_{7}=d D_{6}-G_{6} C_{1}+G_{4} C_{3}-G_{2} C_{5},
$$

respectively. The corresponding gauge-invariant $\mathrm{WZ}$ term is given by

$$
\mathcal{L}_{\mathrm{WZ}}=D_{6}-\mathcal{G}_{5} C_{1}+\mathcal{G}_{3} C_{3}-\mathcal{G}_{1} C_{5} \equiv \mathcal{D}_{6},
$$

which contains the worldvolume potentials $c_{0}, c_{2}$ and $c_{4}$. Imposing a duality relation between them we are left with a scalar and a self-dual 2 -form. Together with the 4 embedding scalars these form the bosonic sector of an $8+8$ tensor multiplet in the six-dimensional worldvolume. The extra $c_{0}$ scalar indicates that this solitonic 5-brane has an 11-dimensional origin as the M5-brane.

We next consider the 8 -form $D_{8}$. Like in the IIB case $D_{8}$ transforms to $H_{3}$ under a solitonic gauge transformation. Furthermore, the transformation rules and curvature contain a free real parameter $\alpha$ :

$$
\delta D_{8}=d \Lambda_{7}+\alpha G_{8} \lambda_{0}+(1-\alpha) G_{6} \lambda_{2}-(2-\alpha) G_{4} \lambda_{4}+(3-\alpha) G_{2} \lambda_{6}+H_{3} \Lambda_{5}
$$

and

$$
F_{9}=d D_{8}-\alpha G_{8} C_{1}-(1-\alpha) G_{6} C_{3}+(2-\alpha) G_{4} C_{5}-(3-\alpha) G_{2} C_{7}+H_{3} D_{6} .
$$

As in the IIB case the presence of the free parameter $\alpha$ is related to the field redefinition

$$
D_{8} \rightarrow D_{8}+\alpha\left(C_{1} C_{7}-C_{3} C_{5}\right) .
$$

This a consistent redefinition due to the fact that

$$
d\left(C_{1} C_{7}-C_{3} C_{5}\right)=G_{2} C_{7}-G_{4} C_{5}+G_{6} C_{3}-G_{8} C_{1} .
$$

The gauge-invariant WZ term is given by

$$
\mathcal{L}_{\mathrm{WZ}}=D_{8}-\alpha \mathcal{G}_{7} C_{1}-(1-\alpha) \mathcal{G}_{5} C_{3}+(2-\alpha) \mathcal{G}_{3} C_{5}-(3-\alpha) \mathcal{G}_{1} C_{7}+\mathcal{F}_{2} \mathcal{D}_{6} \equiv \mathcal{D}_{8}+\mathcal{F}_{2} \mathcal{D}_{6}
$$

which contains the worldvolume fields $c_{0}, c_{2}, c_{4}, c_{6}$ and $b_{1}$. Imposing a duality relation on the $c$ fields we are left with a scalar, a (non-self-dual) 2-form and a vector. For no choice of $\alpha$ can we get rid of both the scalar and the 2-form and therefore these fields will not fit into a vector multiplet. As in the IIB case, there is a choice of $\alpha$ for which the gauge transformations look the most symmetric. This choice is $\alpha=3 / 2$, which gives

$$
\delta D_{8}=d \Lambda_{7}+\frac{3}{2} G_{8} \lambda_{0}-\frac{1}{2} G_{6} \lambda_{2}-\frac{1}{2} G_{4} \lambda_{4}+\frac{3}{2} G_{2} \lambda_{6}+H_{3} \Lambda_{5} .
$$


Finally, we consider the two 10-form potentials. In this case one obtains that the transformations depend on a real parameter $\beta$, and in particular using eq. (3.28) one gets

$$
\begin{aligned}
\delta D_{10}= & d \Lambda_{9}+\beta G_{10} \lambda_{0}+\left(\frac{3}{2}-\beta\right) G_{8} \lambda_{2}-(2-\beta) G_{6} \lambda_{4}+\left(\frac{3}{2}-\beta\right) G_{4} \lambda_{6} \\
& +\beta G_{2} \lambda_{8}+H_{3} \Lambda_{7} .
\end{aligned}
$$

Given that no field redefinition is possible, the parameter $\beta$ signals the presence of two independent 10-forms. The corresponding gauge-invariant WZ term is given by

$$
\begin{aligned}
\mathcal{L}_{\mathrm{WZ}}= & D_{10}-\beta \mathcal{G}_{9} C_{1}-\left(\frac{3}{2}-\beta\right) \mathcal{G}_{7} C_{3}+(2-\beta) \mathcal{G}_{5} C_{5}-\left(\frac{3}{2}-\beta\right) \mathcal{G}_{3} C_{7}-\beta \mathcal{G}_{1} C_{9} \\
& +\mathcal{F}_{2} \mathcal{D}_{8}+\frac{1}{2} \mathcal{F}_{2} \mathcal{F}_{2} \mathcal{D}_{6}
\end{aligned}
$$

which contains the potentials $c_{0}, c_{2}, c_{4}, c_{6}, c_{8}$ and $b_{1}$. This corresponds to a scalar multiplied by $\beta$, a 2 -form multiplied by $\frac{3}{2}-\beta$, a self-dual 4 -form multiplied by $2-\beta$ plus a vector, and for no choice of the parameter $\beta$ one obtains the bosonic sector of a ten-dimensional vector multiplet.

\section{Gauge algebra of solitonic fields in any dimension}

In this section we want to determine the gauge algebra of the fields in table 2. In [10] the gauge algebra of the $\alpha=0$ and $\alpha=-1$ fields in any dimension was considered. Because of conservation of the $\mathbb{R}^{+}$weight, the $\alpha=-2$ fields, which are the $D$ fields, transform among themselves and into the $B$ fields and $C$ fields of [10], but not into fields with lower $\alpha$. In order to proceed, we first review the results and the notation of [10]. The $\alpha=0$ fields are $B_{1, A}$ and $B_{2}$, and their corresponding gauge transformations and fields strengths are

$$
\begin{aligned}
\delta B_{1, A} & =d \Sigma_{0, A} \\
\delta B_{2} & =d \Sigma_{1}-H_{2, A} \Sigma_{0}^{A}
\end{aligned}
$$

and

$$
\begin{aligned}
H_{2, A} & =d B_{1, A} \\
H_{3} & =d B_{2}+H_{2, A} B_{1}^{A} .
\end{aligned}
$$

This implies the Bianchi identity

$$
d H_{3}=H_{2, A} H_{2}^{A} .
$$

The $\alpha=-1$ fields $C$ are spinors of $\mathrm{SO}(d, d)$, of plus or minus chirality according to whether the form has odd or even rank. That is, following the notation explained in appendix A,

$$
C_{2 n+1, \alpha}=\left(\begin{array}{c}
C_{2 n+1, a} \\
0
\end{array}\right), \quad C_{2 n, \alpha}=\left(\begin{array}{c}
0 \\
C_{2 n, \dot{a}}
\end{array}\right) .
$$

Similarly, the gauge parameters are

$$
\lambda_{2 n, \alpha}=\left(\begin{array}{c}
\lambda_{2 n, a} \\
0
\end{array}\right), \quad \lambda_{2 n-1, \alpha}=\left(\begin{array}{c}
0 \\
\lambda_{2 n-1, \dot{a}}
\end{array}\right) .
$$


The gauge transformations and field strengths are

$$
\delta C_{n, \alpha}=d \lambda_{n-1, \alpha}+H_{3} \lambda_{n-3, \alpha}-H_{2, A} \Gamma_{\alpha}^{A \beta} \lambda_{n-2, \beta}
$$

and

$$
G_{n+1, \alpha}=d C_{n, \alpha}+H_{3} C_{n-2, \alpha}+H_{2, A} \Gamma_{\alpha}^{A \beta} C_{n-1, \beta}
$$

This implies

$$
d G_{n+1, \alpha}=-H_{3} G_{n-1, \alpha}+H_{2, A} \Gamma_{\alpha}^{A \beta} G_{n, \beta} .
$$

In the following we will consider bilinears made out of these $\mathrm{SO}(d, d)$ spinors. We thus define $\bar{G}$ as

$$
\bar{G}^{\alpha}=i G_{\beta} C^{\beta \alpha} \text { for } d=2,3 \quad \bar{G}^{\alpha}=G_{\beta} C^{\beta \alpha} \text { for } d=1,4,5,
$$

where $C^{\alpha \beta}$ is the charge conjugation matrix, whose properties for all $d$ are described in appendix A. In the following we will also need the relations

$$
\bar{G}_{n+1}^{\alpha}=d \bar{C}_{n}^{\alpha}+H_{3} \bar{C}_{n-2}^{\alpha}+H_{2, A} \bar{C}_{n-1}^{\beta} \Gamma_{\beta}^{A \alpha}
$$

and

$$
d \bar{G}_{n+1}^{\alpha}=-H_{3} \bar{G}_{n-1}^{\alpha}+H_{2, A} \bar{G}_{n}^{\beta} \Gamma_{\beta}^{A \alpha}
$$

where we have made use of eq. (A.20). The bilinears in our expressions will always have all their spinor indices contracted, and we will always write them without indices, so that the contraction will always be understood.

We now proceed with deriving the gauge algebra of the $D$ fields, which are listed in table 2. We denote the gauge parameters of the $D$ fields with $\Lambda$, and the field strengths with $F$. We want to determine the gauge algebra of the $D$ fields in the abelian basis, that is we require that the gauge transformations only contain gauge invariant quantities. Up to field redefinitions, the gauge transformations can then always be written schematically as $\delta D=d \Lambda+\bar{G} \Gamma \lambda+H \Lambda$, where here $\Gamma$ schematically denotes the antisymmetric product of a number of gamma matrices equal to the number of $\mathrm{SO}(d, d)$ indices of the field $D$. Before one determines the gauge transformations of the $D$ fields, one has to be sure that the abelian basis that one chooses is not redundant, that is that there are no field redefinitions within the basis. What we are looking after is the possibility that a bilinear $\bar{C} \Gamma C$ in the $C$ fields is such that $d(\bar{C} \Gamma C)=G \Gamma C$. When this occurs, we have to subtract this to the number of $D$ fields that close the algebra in the abelian basis to get the actual counting right. We want to determine an $m$-form given by the combination

$$
\sum_{n=1}^{\left[\frac{m}{2}\right]} \alpha_{n} \bar{C}_{m-n} \Gamma_{A_{1} \ldots A_{p}} C_{n}
$$

such that when computing its curl all terms of the form $H \bar{C} \Gamma C$ vanish. Given the chirality of the fields and the form of the $C$ matrix in any $d$, given in eq. (A.8), the only possibility is that $m+p+D$ must be even. We first consider the case $p=0$, that is the case of a 
T-duality singlet. By direct inspection, one can show that in any $D$ there is a solution for $m=D-2$, and the whole set of solutions is

$$
\begin{array}{lll}
m=2: & \bar{C}_{1} C_{1} & (D=4,8) \\
m=3: & \bar{C}_{2} C_{1} & (D=5,9) \\
m=4: & \bar{C}_{3} C_{1}-\frac{1}{2} \bar{C}_{2} C_{2} & (D=6) \\
m=5: & \bar{C}_{4} C_{1}+\bar{C}_{3} C_{2} & (D=7) \\
m=6: & \bar{C}_{5} C_{1}-\bar{C}_{4} C_{2}-\frac{1}{2} \bar{C}_{3} C_{3} & (D=8) \\
m=7: & \bar{C}_{6} C_{1}+\bar{C}_{5} C_{2}-\bar{C}_{4} C_{3} & (D=9)
\end{array}
$$

One can then show that for $p \neq 0$ there are no solutions. To summarise, this analysis shows that this field redefinition will only affect the counting of the $D-2$-forms that are singlets of $\mathrm{SO}(d, d)$. By looking at table 2, we expect to find a single field $D_{D-2}$ after this field redefinition is taken into account. As we will see, this will be confirmed by our explicit computations.

We now determine the gauge algebra of the fields given in table 2 . We start by analysing the fields with the highest amount of T-duality indices for each rank. These fields are

$$
D_{D-4+m, A_{1} \ldots A_{m}} \quad m=0,1, \ldots, 4
$$

and one can see that the redundancy of eq. (4.13) does not affect these fields. We write the gauge transformation of these fields as

$$
\begin{aligned}
\delta D_{D-4+m, A_{1} \ldots A_{m}}= & d \Lambda_{D-5+m, A_{1} \ldots A_{m}}+\sum_{n=0}^{D-6+m} a_{n}^{(m)} \bar{G}_{D-4+m-n} \Gamma_{A_{1} \ldots A_{m}} \lambda_{n} \\
& -m H_{2,\left[A_{1}\right.} \Lambda_{\left.D-6+m, A_{2} \ldots A_{m}\right]}
\end{aligned}
$$

and the corresponding field strength as

$$
\begin{aligned}
F_{D-3+m, A_{1} \ldots A_{m}}= & d D_{D-4+m, A_{1} \ldots A_{m}}+\sum_{n=0}^{D-6+m} b_{n}^{(m)} \bar{G}_{D-4+m-n} \Gamma_{A_{1} \ldots A_{m}} C_{n+1} \\
& +m H_{2,\left[A_{1}\right.} D_{\left.D-5+m, A_{2} \ldots A_{m}\right]} .
\end{aligned}
$$

The gauge invariance with respect to the gauge parameters $\Lambda$ and $\Sigma$ is already implied by the form in which we have written the last term in eqs. (4.15) and (4.16), while the real parameters $a_{n}^{(m)}$ and $b_{n}^{(m)}$ can be determined by imposing gauge invariance of the curvatures $F_{D-3+m, A_{1} \ldots A_{m}}$ with respect to the parameters $\lambda$. Formally, this applies to the $D$-forms as well, although their field strengths are actually identically zero. Using equation (4.11), the reader can see that the variation of $F_{D-3+m, A_{1} \ldots A_{m}}$ produces terms of four different structures, that is the $d \lambda$ terms $\bar{G} \Gamma_{A_{1} \ldots A_{m}} d \lambda$, two types of $H_{2}$ terms $H_{2}^{B} \bar{G} \Gamma_{B A_{1} \ldots A_{m}} \lambda$ and $H_{2,\left[A_{1}\right.} \bar{G} \Gamma_{\left.A_{2} \ldots A_{m}\right]} \lambda$, and finally the $H_{3}$ terms $H_{3} \bar{G} \Gamma_{A_{1} \ldots A_{m}} \lambda$. Putting to zero all of them one 
obtains the equations

$$
\begin{aligned}
a_{n}^{(m)}(-)^{D+m-n}+b_{n}^{(m)} & =0 & & n=0, \ldots, D-6+m \\
a_{n}^{(m)}-b_{n+1}^{(m)}(-)^{m} & =0 & & n=0, \ldots, D-7+m \\
m\left(a_{n}^{(m)}+b_{n+1}^{(m)}(-)^{m}+a_{n}^{(m-1)}\right) & =0 & & n=0, \ldots, D-7+m \\
-a_{n}^{(m)}+b_{n+2}^{(m)}(-)^{D+m-n} & =0 & & n=0, \ldots, D-8+m .
\end{aligned}
$$

Fixing the normalisation so that $a_{0}^{(0)}=1$, the solution of this set of equations is

$$
\begin{aligned}
& a_{0}^{(m)}=\left(-\frac{1}{2}\right)^{m} \\
& a_{1}^{(m)}=\left(-\frac{1}{2}\right)^{m}(-)^{D} \\
& b_{0}^{(m)}=-\left(\frac{1}{2}\right)^{m}(-)^{D} \\
& b_{1}^{(m)}=\left(\frac{1}{2}\right)^{m}
\end{aligned}
$$

while all other values are given by the relation

$$
a_{n+2}^{(m)}+a_{n}^{(m)}=0 \quad b_{n+2}^{(m)}+b_{n}^{(m)}=0 .
$$

This gives the gauge algebra of all the fields in equation (4.14).

Next, we consider the other fields in table 2 , that are $D_{D-2}, D_{D-1, A}$ and $D_{D}, D_{D}^{\prime}$ and $D_{D, A B}$. We first consider the fields

$$
D_{D-2+m, A_{1} \ldots A_{m}} \quad m=0,1,2,
$$

while the $D$-form singlets will be separately discussed later. We write the gauge transformation as

$$
\begin{aligned}
\delta D_{D-2+m, A_{1} \ldots A_{m}}= & d \Lambda_{D-3+m, A_{1} \ldots A_{m}}+\sum_{n=0}^{D-4+m} c_{n}^{(m)} \bar{G}_{D-2+m-n} \Gamma_{A_{1} \ldots A_{m}} \lambda_{n} \\
& +H_{3} \Lambda_{D-5+m, A_{1} \ldots A_{m}}-m H_{2,\left[A_{1}\right.} \Lambda_{\left.D-4+m, A_{2} \ldots A_{m}\right]}-H_{2}^{B} \Lambda_{D-4+m, B A_{1} \ldots A_{m}}
\end{aligned}
$$

while the field strength is

$$
\begin{aligned}
F_{D-1+m, A_{1} \ldots A_{m}}= & d D_{D-2+m, A_{1} \ldots A_{m}}+\sum_{n=0}^{D-4+m} d_{n}^{(m)} \bar{G}_{D-2+m-n} \Gamma_{A_{1} \ldots A_{m}} C_{n+1} \\
& +H_{3} D_{D-4+m, A_{1} \ldots A_{m}}+m H_{2,\left[A_{1}\right.} D_{\left.D-3+m, A_{2} \ldots A_{m}\right]}+H_{2}^{B} D_{D-3+m, B A_{1} \ldots A_{m}}
\end{aligned}
$$

where $c_{n}^{(m)}$ and $d_{n}^{(m)}$ are real parameters. Again, we have written the field strengths and gauge transformations so that gauge invariance of the field strengths with respect to the parameters $\Sigma$ and $\Lambda$ is already implied, while imposing gauge invariance with respect to $\lambda$ leads to the equations

$$
\begin{aligned}
c_{n}^{(m)}(-)^{D+m-n}+d_{n}^{(m)} & =0 & & n=0, \ldots, D-4+m \\
c_{n}^{(m)}-d_{n+1}^{(m)}(-)^{m}+a_{n}^{(m+1)} & =0 & & n=0, \ldots, D-5+m \\
m\left(c_{n}^{(m)}+d_{n+1}^{(m)}(-)^{m}+c_{n}^{(m-1)}\right) & =0 & & n=0, \ldots, D-5+m \\
-c_{n}^{(m)}+d_{n+2}^{(m)}(-)^{D+m-n}+a_{n}^{(m)} & =0 & & n=0, \ldots, D-6+m .
\end{aligned}
$$


The reader can realise by direct inspection that there is a one parameter family of solutions in each dimension. This is expected from eq. (4.13), which shows that in each dimension one can add to the $(D-2)$-form a suitable combination quadratic in the $C$ fields such that the gauge transformation of the resulting field is still in the abelian basis. Taking into account this redundancy, one finds the solutions corresponding to the $D_{D-2+m}, A_{1} \ldots A_{m}$ as in table 2 .

We consider explicitly here as a prototype example the five-dimensional case. Denoting with $\alpha$ the undetermined parameter, we have that the algebra of the $D_{3}$ form closes if

$$
\begin{array}{ll}
c_{0}^{(0)}=\alpha & c_{1}^{(0)}=-\alpha+\frac{1}{2} \\
d_{0}^{(0)}=\alpha & d_{1}^{(0)}=\alpha-\frac{1}{2} .
\end{array}
$$

We choose to fix the value of $\alpha$ such that the solution looks the most symmetric. This value is $\alpha=\frac{1}{4}$, as can be seen from eq. (4.24). This gives

$$
\begin{array}{ll}
c_{0}^{(0)}=\frac{1}{4} & c_{1}^{(0)}=\frac{1}{4} \\
d_{0}^{(0)}=\frac{1}{4} & d_{1}^{(0)}=-\frac{1}{4} .
\end{array}
$$

Plugging this solution back in equation (4.23) one then finds

$$
\begin{array}{lll}
c_{0}^{(1)}=-\frac{1}{4} & c_{1}^{(1)}=0 & c_{2}^{(1)}=-\frac{1}{4} \\
d_{0}^{(1)}=\frac{1}{4} & d_{1}^{(1)}=0 & d_{2}^{(1)}=\frac{1}{4}
\end{array}
$$

for the $D-1$-form and

$$
\begin{array}{llll}
c_{0}^{(2)}=\frac{3}{16} & c_{1}^{(2)}=-\frac{1}{16} & c_{2}^{(2)}=\frac{1}{16} & c_{3}^{(2)}=-\frac{3}{16} \\
d_{0}^{(2)}=\frac{3}{16} & d_{1}^{(2)}=\frac{1}{16} & d_{2}^{(2)}=\frac{1}{16} & d_{3}^{(2)}=\frac{3}{16}
\end{array}
$$

for the $D$-form. Similarly, it is straightforward to derive the solutions of eq. (4.23) in other dimensions.

Finally, we consider the two $D$-form singlets in table 2 . We write the gauge transformations and the fields strength (again, the gauge transformations for these fields can be determined imposing the formal gauge invariance of a field strength, although this is actually identically zero) as

$$
\delta D_{D}=d \Lambda_{D-1}+\sum_{n=0}^{D-2} e_{n} \bar{G}_{D-n} \lambda_{n}+H_{3} \Lambda_{D-3}-H_{2}^{A} \Lambda_{D-2, A}
$$

and

$$
F_{D+1}=d D_{D}+\sum_{n=0}^{D-2} f_{n} \bar{G}_{D-n} C_{n+1}+H_{3} D_{D-2}+H_{2}^{A} D_{D-1, A},
$$

with $e_{n}$ and $f_{n}$ real parameters, and gauge invariance with respect to $\Sigma$ and $\Lambda$ already implied. Imposing gauge invariance with respect to $\lambda$ leads to the equations

$$
\begin{array}{rlrl}
e_{n}(-)^{D-n}+f_{n}=0 & & n=0, \ldots, D-2 \\
e_{n}-f_{n+1}+c_{n}^{(1)}=0 & & n=0, \ldots, D-3 \\
-e_{n}+f_{n+2}(-)^{D-n}+c_{n}^{(0)} & =0 & & n=0, \ldots, D-4 .
\end{array}
$$


The reader can verify by directly solving the equations that in each dimension there are two solutions. Given that there are no possible field redefinitions, as shown in eq. (4.13), this implies that there are two $D$-form singlets, in agreement with table 2 . As an example, we give again the solution for the five-dimensional case, using the solution (4.25) and (4.26) for the coefficients $c_{n}^{(0)}$ and $c_{n}^{(1)}$. That is

$$
\begin{array}{llll}
e_{0}=\beta & e_{1}=-\beta+\frac{1}{4} & e_{2}=-\beta+\frac{1}{4} & e_{3}=\beta \\
f_{0}=\beta & f_{1}=\beta-\frac{1}{4} & f_{2}=-\beta+\frac{1}{4} & f_{3}=-\beta .
\end{array}
$$

This solution contains an arbitrary real parameter $\beta$, which is the relative normalisation of the two $D$-forms.

To conclude, we have determined the gauge transformations of all the fields in table 2 for any dimension. In the next section we will use these results to determine the corresponding WZ terms, and by analysing the world-volume field content we will show in which cases we expect these WZ terms to be describing supersymmetric branes.

\section{$5 \quad$ String solitons in any dimension}

The aim of this section is to derive a universal expression for the WZ terms that we claim to describe the charge sector of the effective action of string solitons in any dimension. This analysis is the generalisation to any dimension of the one performed in section 3 in the ten-dimensional case. For any of the forms given in table 2, whose gauge transformations have been determined in the previous section, one can introduce suitable world-volume fields that lead to a gauge invariant WZ term. We expect the world-volume fields to occur in a democratic formulation together with their electromagnetic duals. We stress that this is a world-volume duality relating fields in the effective action, which has nothing to do with the target space electromagnetic duality relating the various bulk fields in $D$ dimension. After this electromagnetic duality is taken into account, we expect the worldvolume fields of supersymmetric branes to belong to half-supersymmetry multiplets. These multiplets are vector multiplets in all cases, with the exception of the 5-branes, for which either a vector multiplet or a tensor multiplet can occur. This will thus be our selection criterion, we select all WZ terms whose corresponding world volume fields fit within a half-supersymmetry multiplet.

We first review how the D-brane WZ terms in [10] were constructed. Given that the gauge algebra reviewed in eqs. (4.1) and (4.6) only contains the field strengths of the $\alpha=0$ fields, one only needs to introduce world-volume fields $b_{0, A}$ and $b_{1}$ associated to these fields, that is

$$
\begin{aligned}
b_{0, A}: & \delta b_{0, A} & =-\Sigma_{0, A} \\
b_{1}: & \delta b_{1} & =-\Sigma_{1},
\end{aligned}
$$

and define the gauge invariant quantities

$$
\begin{aligned}
\mathcal{F}_{1, A} & =d b_{0, A}+B_{1, A} \\
\mathcal{F}_{2} & =d b_{1}+B_{2}-H_{2, A} b_{0}^{A} .
\end{aligned}
$$

This leads to the gauge-invariant WZ terms in eqs. (1.5) and (1.8). 
It is worth showing how eq. (1.8) leads to the correct number of worldvolume degrees of freedom for any $p$-dimensional worldvolume in $D$ dimensions. This analysis was not performed in full detail in [10]. The WZ term (1.8) contains the worldvolume vector $b_{1}$ together with $2 d$ worldvolume scalars $b_{0, A}$. The vector corresponds to $p-2$ degrees of freedom, and considering also the $D-p$ embedding scalars one needs $d$ additional worldvolume scalars to fill the bosonic sector of a vector multiplet in $p$ dimensions, while there are $2 d$ scalar fields $b_{0, A}$, that is twice as many. We now show that in eq. (1.8) only half of the $b_{0, A}$ fields occur. To understand the mechanism, it is enough to expand (1.8) and consider only the first $\mathcal{F}_{1, A}$ term, that is

$$
C_{p, \alpha}+\mathcal{F}_{1, A} \Gamma_{\alpha}^{A \beta} C_{p-1, \beta}+\ldots
$$

We move to an $\mathrm{SO}(d, d)$ lightcone basis, and we denote the light-cone directions as $1 \pm$, $2 \pm, \ldots, d \pm$. The analysis of the properties of the $\mathrm{SO}(d, d)$ Gamma matrices in the lightcone basis is performed in detail in appendix A. For a given value of the spinor index $\alpha$, one can show that for any fixed $n=1, \ldots, d$ only one of the two matrices $\Gamma_{n \pm, \alpha}{ }^{\beta}$ gives a non-zero result when acting on a chiral spinor. This can be seen explicitly in the basis chosen in appendix A, see e.g. eqs. (A.24), (A.26) or (A.27) and (A.28), and one can show using the $\mathrm{SO}(d, d)$ Gamma matrices given in (A.10) and (A.11) and the lightcone ones given in (A.22) that this result is completely general. This analysis proves that for any given D-brane only half of the $2 d$ worldvolume scalars actually occurs, and this results in the correct number of degrees of freedom for a $p$-dimensional worldvolume vector multiplet.

We next consider the $D$ fields, whose gauge algebra has been derived in the previous section. The corresponding gauge transformations contain both the field strengths of the $B$ and of the $C$ fields, which implies that in order to write down a gauge invariant WZ term one has to introduce, together with the world-volume fields in (5.1), the world-volume fields $c_{n, \alpha}$ such that

$$
\delta c_{n, \alpha}=-\lambda_{n, \alpha} .
$$

This implies that these fields are $\mathrm{SO}(d, d)$ spinors of one chirality for $n$ even and of opposite chirality for $n$ odd,

$$
c_{2 n, \alpha}=\left(\begin{array}{c}
c_{2 n, a} \\
0
\end{array}\right), \quad c_{2 n-1, \alpha}=\left(\begin{array}{c}
0 \\
c_{2 n-1, \dot{a}}
\end{array}\right) .
$$

One then introduces the gauge invariant world-volume curvatures

$$
\mathcal{G}_{n, \alpha}=d c_{n-1, \alpha}+C_{n, \alpha}-H_{2, A} \Gamma_{\alpha}^{A \beta} c_{n-2, \beta}+H_{3} c_{n-3, \alpha},
$$

whose curl is

$$
d \mathcal{G}_{n, \alpha}=G_{n+1, \alpha}-H_{2, A} \Gamma_{\alpha}^{A \beta} \mathcal{G}_{n-1, \beta}-H_{3} \mathcal{G}_{n-2, \alpha} .
$$

Using this, one can define a gauge invariant WZ term in all cases.

We first consider the fields $D_{D-4+m, A_{1} \ldots A_{m}}$ in eq. (4.14), whose gauge transformation is given in eq. (4.15), with the coefficients given in eqs. (4.18) and (4.19). The corresponding 
WZ term is given by the universal expression

$$
\left[e^{\mathcal{F}_{1}} \frac{m !}{\tilde{m} !}\left(D_{D-4+\tilde{m}}-\sum_{n=0}^{D-6+\tilde{m}} a_{n}^{(\tilde{m})}(-)^{D+\tilde{m}-n} \overline{\mathcal{G}}_{D-5+\tilde{m}-n} \Gamma_{(\tilde{m})} C_{n+1}\right)\right]_{D-4+m, A_{1} \ldots A_{m}} .
$$

The rule in this expression is that we have to pick a $D-4+m$ form, so for any $D-4+\tilde{m}$-form in the term in curved brackets we have to pick $m-\tilde{m}$ powers of $\mathcal{F}_{1}$. The $\mathrm{SO}(d, d)$ indices are always meant to be antisymmetrised, and we have denoted with $\Gamma_{(\tilde{m})}$ the antisymmetric product of $\tilde{m}$ Gamma matrices. The real coefficients $a_{n}^{(\tilde{m})}$ are given in eqs. (4.18) and (4.19). Using eqs. (4.6) and (4.15), as well as eq. (5.7), one can show that the variation of eq. (5.8) is a total derivative.

The $c_{n, \alpha}$ fields that appear in eq. (5.8) are $c_{0, \alpha}, c_{1, \alpha}, \ldots, c_{D-6+m, \alpha}$. We will impose in all cases that the $c$ fields are related by electromagnetic duality relations on the $D-4+m$ dimensional world-volume, which implies that the field $c_{n, \alpha}$ and the field $c_{D-6+m-n, \alpha}$ are duals. When $D-4+m$ is even, the field of rank $(D-6+m) / 2$ satisfies a self-duality condition. The reason for assuming this is that we have already shown in section 3 that this leads to the right counting of degrees of freedom in ten dimensions. When counting the bosonic world-volume degrees of freedom, one has also to consider the $4-m$ embedding scalars, together with the extra scalars $b_{0, A}$. We are counting the degrees of freedom for fixed $A_{1} \ldots A_{m}$, where the indices are all different because of antisymmetry. This implies that the scalars $b_{0, A}$ contribute as $m$ degrees of freedom. Therefore, the embedding scalars plus the scalars $b_{0, A}$ contribute in total as four degrees of freedom in all cases.

We then count the degrees of freedom carried by the $c$ fields. We recall that the $\mathrm{SO}(d, d)$ chiral spinors $c_{n, \alpha}$ have $2^{d-1}$ real components. As we will show in a case by case analysis in the following, one needs $2^{d-m-1}$ chiral components to get the right counting of the degrees of freedom in each case. When $m=1$ the different light-cone $\mathrm{SO}(d, d)$ directions correspond to independent branes. This is because, as shown in appendix A, the light-cone Gamma matrices have the property of projecting out half of the components of a spinor for each chirality. As already discussed, we denote the light-cone directions as $1 \pm, 2 \pm, \ldots, d \pm$. For $m=2, m=3$ and $m=4$ the request that the degrees of freedom collect in a multiplet, that is the request that the product of $m$ Gamma matrices projects a chiral spinor to $2^{d-m-1}$ chiral components, imposes that one has to consider only the components corresponding to indices $n_{1} \pm n_{2} \pm \ldots n_{m} \pm$, with the $n_{i}$ all different. In formula, we have

$$
\Gamma_{(m)} \rightarrow \Gamma_{n_{1} \pm n_{2} \pm \ldots n_{m} \pm}, \quad \text { all } n_{i} \text { different. }
$$

As discussed in appendix A, this forms a conjugacy class, and the corresponding products of $m$ Gamma matrices satisfy the property of being nilpotent. In $D=9, \ldots, 6$ one has among the rest the case $m=d$, which is special because in this case the Gamma matrices with indices $n_{1} \pm \ldots n_{d} \pm$ ( $n$ 's all different) map a chiral spinor to either zero or one component (according to the chirality of the spinor). Given these rules, the outcome of our analysis will be that eq. (5.8) leads to a world-volume vector multiplet for $m<d$, and to either a vector or a tensor multiplet for $m=d$ (which corresponds to a 5-brane), depending on 


\begin{tabular}{|c|c|c|c|c|c|c|}
\hline$D$ & \multicolumn{2}{|c|}{$m=2$} & \multicolumn{2}{c|}{$m=3$} & \multicolumn{2}{c|}{$m=4$} \\
\hline 8 & $\mathbf{3}^{+}+\mathbf{3}^{-}$ & $2+2$ & & & & \\
\hline 7 & $\mathbf{1 5}$ & 12 & $\mathbf{1 0}^{+}+\mathbf{1 0}^{-}$ & $4+4$ & & \\
\hline 6 & $\mathbf{2 8}$ & 24 & $\mathbf{5 6}$ & 32 & $\mathbf{3 5}^{+}+\mathbf{3 5}^{-}$ & $8+8$ \\
\hline 5 & $\mathbf{4 5}$ & 40 & $\mathbf{1 2 0}$ & 80 & $\mathbf{2 1 0}$ & 80 \\
\hline
\end{tabular}

Table 9. Table giving the dimension of the conjugacy classes of the solitonic branes in various dimensions. For each $m$, we denote in the first column the $\mathrm{SO}(d, d)$ representation of the field $D_{D-4+m, A_{1} \ldots A_{m}}$, whose dimension is $\left(\begin{array}{c}2 d \\ m\end{array}\right)$, and in the second column the dimension of the conjugacy class, $2^{m}\left(\begin{array}{c}d \\ m\end{array}\right)$. For $d=m$ the representation splits in selfdual and anti-selfdual, corresponding to branes supporting either a vector or a tensor multiplet.

whether one takes the self-dual or the anti-self-dual part of the completely antisymmetric $\mathrm{SO}(d, d)$ representation with $d$ indices. We will also see that one cannot obtain the right degrees of freedom for $m>d$, so we expect this case not to correspond to a brane. This result is summarised in table 1 . The branes are along the light-cone directions described above, and their number for each dimension and for each $m$ is given in table 9 (see also appendix A for the details).

We now analyse the explicit form of eq. (5.8) in all the specific cases, that is for all the different values of $m$ from 0 to 4 . The fact that the counting works for the case $m=0$ actually already guarantees that it has to work for higher $m$. For instance, one gets for $m=1$ in $D$ dimensions exactly the same counting as for $m=0$ in $D+1$ dimensions. The reason is that when going from $D+1$ to $D$ dimensions, the number of spinor components for each $c$ field doubles, which is compensated by the fact that each light-cone Gamma matrix projects out half of the components. This gives the same counting for a $D$-3-dimensional world-volume. The same reasoning applies to the other values of $m$.

$\boldsymbol{m}=\mathbf{0}$. For $m=0$, eq. (5.8) collapses to the expression

$$
D_{D-4}-\sum_{n=0}^{D-6} a_{n}^{(0)}(-)^{D-n} \overline{\mathcal{G}}_{D-5-n} C_{n+1}
$$

containing the world volume fields $c_{0, \alpha}, \ldots, c_{D-6, \alpha}$, with $c_{n, \alpha}$ dual to $c_{D-6-n, \alpha}$. In addition to these fields, one has to consider four embedding scalars in any dimension. We now analyse this in different dimensions.

The ten-dimensional case has already been discussed in section 3. We have shown that in the IIA case the fields are $c_{0}, c_{2}$ and $c_{4}$. Imposing duality, one obtains one scalar plus one self-dual tensor, that together with the four embedding scalars makes the bosonic field content of a tensor multiplet in six dimensions. This is the WZ term of an NS5A brane. In the IIB case, instead, one has $c_{1}$ and $c_{3}$, which corresponds to a vector, and together with the four embedding scalars one obtains a vector multiplet, which is the WZ term of an NS5B brane.

In nine dimensions one has a five-dimensional world volume. The world-volume field content is $c_{0}, c_{1}, c_{2}$ and $c_{3}$ (there are no indices because the chiral spinors of $\mathrm{SO}(1,1)$ are 
one-dimensional). Imposing duality, one obtains a vector and a scalar, that together with the four embedding scalars makes a vector multiplet in five dimensions. We thus expect this to be the WZ term of a 4-brane.

In eight dimensions the world-volume is four-dimensional, and the fields are $c_{0, a}, c_{1, \dot{a}}$ and $c_{2, a}$, two-dimensional spinors of the T-duality group $\mathrm{SO}(2,2)$. After duality, this corresponds to two scalars and one vector, which together with the four embedding scalars makes a vector multiplet in four dimensions. We consider this the WZ term of a 3-brane in eight dimensions.

In seven dimensions one has a three-dimensional world-volume, with fields $c_{0, a}$ and $c_{1, \dot{a}}, a, \dot{a}=1, \ldots, 4$. These fields are dual in three dimensions, and together with the four embedding scalars this makes the eight bosonic degrees of freedom of a three-dimensional scalar multiplet. Similarly, in six dimensions one has a two-dimensional 8-component scalar $c_{0, a}$ which satisfies a self-duality condition, that is four degrees of freedom. Again, together with the embedding scalars this sums up to eight bosonic degrees of freedom.

To summarise, we thus expect in all cases the WZ term of eq. (5.10) to correspond to a supersymmetric $D-5$-brane.

$\boldsymbol{m}=1$. Eq. (5.8), evaluated for $m=1$, gives

$$
\begin{aligned}
& D_{D-3, A}+\sum_{n=0}^{D-5} a_{n}^{(1)}(-)^{D-n} \overline{\mathcal{G}}_{D-4-n} \Gamma_{A} C_{n+1} \\
& \quad+\mathcal{F}_{1, A}\left(D_{D-4}-\sum_{n=0}^{D-6} a_{n}^{(0)}(-)^{D-n} \overline{\mathcal{G}}_{D-5-n} C_{n+1}\right) .
\end{aligned}
$$

Clearly, this expression only makes sense in dimension lower than ten. To count the expected degrees of freedom, one has to split the $A$ index in light-cone coordinates, and each light-cone coordinate corresponds to $2^{d-2}$ components for the chiral spinor.

In nine dimensions (that is for $d=1$ ) each of the two light-cone coordinates project on either one chirality or the opposite. Indeed, for $\mathrm{SO}(1,1)$ splitting $\Gamma_{A}$ in light-cone coordinates corresponds to taking the self-dual or the anti-self-dual combination (see appendix A). This means that in the anti-selfdual case one gets $c_{0}, c_{2}$ and $c_{4}$, and in the selfdual case one gets $c_{1}$ and $c_{3}$, in both cases together with 4 additional scalars. Imposing duality one gets a 5-brane supporting a tensor multiplet in the former case and a vector multiplet in the latter.

In eight dimensions each light-cone component selects a single spinor component out of the two components for each chirality. This leaves the fields $c_{0}, c_{1}, c_{2}$ and $c_{3}$ in a five-dimensional world-volume. Imposing duality, this leads to a vector multiplet for each light-cone component. This shows how the halving of the number of spinor components with respect to the $m=0$ case is crucial to get the right counting.

In seven dimensions each light-cone Gamma matrix projects on two components out of the four spinor components for each chirality. This gives two $c_{0}$ 's, two $c_{1}$ 's and two $c_{2}$ 's. Imposing self-duality on the four-dimensional world-volume one gets a vector and two scalars, that together with the additional four scalars makes the bosonic sector of a vector multiplet in four dimensions. 
The same applies to the lower dimensional case. In general, as already stressed, in each dimension $D$ the counting of the degrees of freedom of the $m=1$ branes is the same as the counting of the degrees of freedom of the $m=0$ branes in $D+1$ dimensions.

$\boldsymbol{m}=\mathbf{2}$. In this case in nine dimensions one would expect a 6 -brane, that is a 7 dimensional world-volume, but there is no way of selecting the fields of a seven-dimensional vector multiplet, because there is no way of getting rid of the world-volume fields $c_{2}$ and $c_{3}$, which would correspond to a seven-dimensional rank-2 tensor. We thus expect this not to correspond to a brane. This applies to all the other cases $m>d$.

In eight dimensions, we take the light-cone directions $1 \pm 2 \pm$. For each of these four components one selects out of a 2-component chiral spinor either one component or zero, according to the chirality. The directions $1+2+$ and $1-2-$ select $c_{1}$ and $c_{3}$, while the directions $1+2-$ and $1-2+$ select $c_{0}, c_{2}$ and $c_{4}$. In the first case one gets a vector multiplet, and in the second a tensor multiplet. Therefore, there are two vector branes and two tensor branes, as shown in table 9 .

In seven dimensions, out of the $15 \mathrm{SO}(d, d)$ components of $D_{5, A B}$, one selects the 12 components $1 \pm 2 \pm, 1 \pm 3 \pm$ and $2 \pm 3 \pm$. The products of Gamma matrices in these directions select a single components out of the four-component spinors $c_{n, \alpha}$, resulting in a vector multiplet in a five-dimensional world-volume. All the lower-dimensional cases are analogous, one obtains in $D$ dimensions the same counting of the case $m=1$ in $D+1$ dimensions.

$\boldsymbol{m}=\mathbf{3}$. This case does not exist in nine dimensions, while in eight dimensions it does not lead to a vector multiplet, exactly like the $m=2$ case in nine dimensions. In seven dimensions one considers the directions $1 \pm 2 \pm 3 \pm$, which is eight components. Four of the corresponding Gamma matrices project on a single component of one chirality, and the other four of a single component of the opposite chirality. This gives a six-dimensional world-volume with either a vector or a tensor multiplet.

In lower dimensions $D$ one gets $(D-2)$-branes supporting vector multiplets. These branes correspond to the light-like directions $n_{1} \pm n_{2} \pm n_{3} \pm$, with $n_{1}, n_{2}$ and $n_{3}$ all different, and their number is given in table 9. Again, the counting is precisely the same as the one of the previous case $m=2$ in $D+1$ dimensions.

$\boldsymbol{m}=4$. In this case the branes are spacetime-filling. One does not get a vector multiplet in eight and seven dimensions, while in six dimensions one takes the 16 components $1 \pm 2 \pm$ $3 \pm 4 \pm$. The corresponding products of Gamma matrices project on a single component of a given chirality. This gives 8 branes supporting a vector multiplet and 8 branes supporting a tensor multiplet.

In lower dimensions $D$ one gets $(D-1)$-branes supporting vector multiplets. These branes correspond to the light-like directions $n_{1} \pm n_{2} \pm n_{3} \pm n_{4} \pm$, with $n_{1}, n_{2}, n_{3}$ and $n_{4}$ all different, and their number is given in table 9. Again, as for all the other cases the counting is precisely the same as the one of the previous case $m=3$ in $D+1$ dimensions.

We finally comment on the possibility that the other fields in table 2 lead to WZ terms that satisfy our criteria. One simple reason why this is not the case is that these WZ term must contain the field strength $\mathcal{F}_{2}$, because the corresponding fields contain $H_{3}$ in 


\begin{tabular}{|c|c|c|c|c|c|c|c|}
\hline$D$ & $R$-symmetry & $n=0$ & $n=1$ & $n=2$ & $n=3$ & $n=4$ & $n=5$ \\
\hline IIA & $\mathbf{1}$ & $\mathbf{1}$ & $\mathbf{1}$ & $\mathbf{1}$ & - & 1 & 1 \\
\hline IIB & $\mathrm{SO}(2)$ & - & $\mathbf{2}$ & - & $\mathbf{1}$ & - & $1^{+}+2^{+}$ \\
\hline 9 & $\mathrm{SO}(2)$ & $\mathbf{1}+\mathbf{2}$ & $\mathbf{2}$ & $\mathbf{1}$ & $\mathbf{1}$ & $1+2$ & \\
\hline 8 & $\mathrm{U}(2)$ & $2 \times \mathbf{3}$ & $\mathbf{3}$ & $2 \times \mathbf{1}$ & $1+3$ & $3^{+}+3^{-}$ & \\
\hline 7 & $\mathrm{Sp}(4)$ & $\mathbf{1 0}$ & $\mathbf{5}$ & $1+5$ & 10 & & \\
\hline 6 & $\mathrm{Sp}(4) \times \mathrm{Sp}(4)$ & $(\mathbf{4}, \mathbf{4})$ & $(1,1)$ & $(4,4)$ & $(\mathbf{1 0}, \mathbf{1})^{+}$ & & \\
& & & $(1,5)$ & & $(\mathbf{1}, \mathbf{1 0})^{-}$ & & \\
\hline 5 & $\mathrm{Sp}(8)$ & $1+27$ & 27 & $\mathbf{3 6}$ & & & \\
\hline 4 & $\mathrm{SU}(8)$ & $28+\overline{28}$ & $\mathbf{6 3}$ & $\mathbf{3 6} \mathbf{6}^{+}+\overline{\mathbf{3 6}}^{-}$ & & & \\
\hline 3 & $\mathrm{SO}(16)$ & $\mathbf{1 2 0}$ & $\mathbf{1 3 5}$ & & & & \\
\hline
\end{tabular}

Table 10. This table indicates the $R$-representations of the $n$-form central charges of $3 \leq D \leq 10$ maximal supergravity. If applicable, we have also indicated the space-time duality of the central charges with a superscript \pm . The central charges that are discussed in the text are indicated in italic.

their field strengths and gauge transformations, differently from the fields $D_{D-4+m, A_{1} \ldots A_{m}}$ in eq. (4.14) (see section 4). This would thus lead to an additional world-volume vector, which would make it impossible to form a world-volume multiplet.

In principle one should also consider the possibility of taking linear combinations of fields in table 2 that belong to the same representation. This is the case for the two 7 -forms in 9 dimensions, that are both $\mathrm{SO}(1,1)$ singlets, for the two 7 -forms in 8 dimensions, that are both $\mathrm{SO}(2,2)$ vectors, and of (some of) the $D$-forms in nine, eight and seven dimensions. We have investigated this possibility, and the outcome of our analysis is that there is no possible linear combination that selects the right degrees of freedom.

To summarise, none of these additional fields give WZ terms that satisfy our criteria, and therefore our final result is the one summarised in tables 1 and 9 .

\section{Central charges}

It is interesting to compare our results with what we know about the central charges of the target space Poincare supersymmetry algebra. These charges are $n$-forms that transform as representations of the $R$-symmetry of the supersymmetry algebra, see table 10 . In general an $n$-form central charge relates to a $p$-brane with $p=n$ or $p=D-n$. The relation between central charges and branes [27] only applies to asymptotically flat branes and, therefore, can only be applied to the solitonic $D_{D-4}$ and $D_{D-3, A}$ fields but not to the higher-form $D$-fields. We therefore only consider $(D-5)$-branes and $(D-4)$-branes implying that we consider only solitonic branes for $D \geq 4$. These particular branes, corresponding to $m=0$ and $m=1$, do not transform in non-trivial conjugacy classes. Below we discuss the different dimensions separately and show how all central charges correspond to the branes we have been discussing in this paper. 
IIA. In the ten-dimensional IIA theory we consider 5-branes and 6-branes which are described by the 5 -form and the dual of the 4 -form central charges, respectively. We first consider the 6 -branes. The IIA theory contains only a Dirichlet 6 -brane with a vector multiplet. This D6-brane is described by (the dual of) the 14 -form central charge. We next consider the 5 -branes which are described by the 5 -form central charges. In the Dbrane sector there are no 5 -branes. In the solitonic sector there is a solitonic 5-brane with a tensor multiplet. Finally, in the gravitational sector there is a KK monopole with a vector multiplet [28]. In total we find two 5-branes, one with a vector and one with a tensor multiplet. These two branes are described by the $\mathbf{1} 5$-form central charge and its dual.

$\boldsymbol{I I B}$. Like in the IIA case, we consider 5-branes and 6-branes which are described by the 5 -form and the dual of the 4-form central charges, respectively. The IIB theory has no 6branes and, indeed there is no 4 -form central charge. We next consider the 5-branes. In the D-brane sector the IIB theory contains a D5-brane with a vector multiplet. In the solitonic sector it contains a solitonic 5-brane with a vector multiplet and in the gravitational sector it contains a KK monopole with tensor multiplet [28]. In total we find three 5-branes. The KK monopole, with the tensor multiplet, is described by the singlet $\mathbf{1}^{+}$self-dual central charge whereas the D5-brane and the solitonic 5-brane, with the vector multiplets, are described by the S-doublet $\mathbf{2}^{+}$of self-dual central charges.

$\boldsymbol{D}=\mathbf{9}$. In $D=9$ dimensions we consider the 4 -branes and 5-branes which are both described by the 4 -form central charges. We first consider the 5 -branes. In the D-brane sector there is a Dirichlet 5-brane with a vector multiplet that transforms as a one-dimensional chiral spinor representation of $\mathrm{SO}(1,1)$. In the soliton sector there is a 2-dimensional $\mathrm{T}$ duality vector of solitons that splits into a tensor soliton and a vector soliton. The tensor soliton corresponds to the $\mathbf{1}$ dual 4-form central charge and the Dirichlet 5-brane together with the vector soliton correspond to the $\mathbf{2}$ dual 4 -form central charge. In the 4-brane case we have in the D-brane sector a Dirichlet 4-brane with a vector multiplet that is a one-dimensional chiral spinor of $\mathrm{SO}(1,1)$ and in the soliton sector a singlet vector soliton. Furthermore, in the gravitational sector we have a KK monopole with a vector multiplet. The KK monopole corresponds to the $\mathbf{1}$ 4-form central charge and the Dirichlet 4-brane together with the vector soliton correspond to the $\mathbf{2}$ 4-form central charge.

$\boldsymbol{D}=\mathbf{8}$. In $D=8$ dimensions we consider the 3 -branes and 4-branes which are described by the 3 -form and 4 -form central charges. From now on all branes have vector multiplets. We first consider the 4-branes. In the D-brane sector there is a two-dimensional T-duality spinor of Dirichlet 4-branes. In the soliton sector there is a 4-dimensional T-duality vector of solitons. In total this gives 64 -branes corresponding to the $6 \mathbf{3}^{+}+\mathbf{3}^{-}$-form central charges where each Dirichlet 4-brane together with 2 solitonic branes form a triplet. We next consider the 3 -branes. In the D-brane sector there is a 2-component T-duality spinor of Dirichlet 3-branes. In the soliton sector there is a singlet solitonic 3-brane and in the gravitational sector there is a single KK monopole. This adds up to 4 branes corresponding to the 43 -form central charges. The KK monopole corresponds to the $\mathbf{1}$ central charge while the 2 Dirichlet branes together with the solitonic brane correspond to the $\mathbf{3}$ central charges. 
$\boldsymbol{D}=7$. In $D=7$ dimensions we consider 2-branes and 3 -branes corresponding to 2form and 3-form central charges. We first consider the 3-branes. From the D-brane sector we have a 4-component T-duality spinor of Dirichlet 3-branes. In the solitonic sector we have a 6-component T-duality vector of solitons. Together, they correspond to the $\mathbf{1 0}$ 3 -form central charges. We next consider the 2-branes. In the D-brane sector we have a 4 component T-duality spinor of Dirichlet 2-branes while we have a singlet solitonic 2-brane and KK monopole. The KK monopole corresponds to the 12 -form central charge while the 4 Dirichlet 2-branes and singlet soliton correspond to the $\mathbf{5} 2$-form central charge.

$\boldsymbol{D}=\mathbf{6}$. In $D=6$ dimensions we consider 1-branes and 2-branes corresponding to the 1form and 2-form central charges. We first consider the 2-branes. We have an 8-component T-duality spinor of Dirichlet 2-branes and an 8-component T-duality vector of string solitons. Together, they correspond to the $16(\mathbf{4}, \mathbf{4}) 2$-form central charges. We next consider the 1-branes. In this case also the fundamental sector contributes with a singlet fundamental string. On top of that we have an 8-component T-duality spinor of Dirichlet 1-branes, a singlet string soliton and a singlet KK monopole adding up to a total of 11 1-branes. The KK monopole corresponds to the $(\mathbf{1}, \mathbf{1})$ 1-form central charge. Four Dirichlet branes together with the fundamental string correspond to the $(\mathbf{5}, \mathbf{1})$ 1-form central charges while the remaining 4 Dirichlet branes together with the string soliton correspond to the $(\mathbf{1}, \mathbf{5})$ 1-form central charges.

$\boldsymbol{D}=\mathbf{5}$. In $D=5$ dimensions we consider 0 -branes and 1-branes corresponding to the 0 -form and 1-form central charges. We first consider the 1-branes. We have a singlet fundamental string, a 16-component T-duality spinor of Dirichlet 1-branes and a 10-component T-duality vector of string solitons. Together, these 27 1-branes correspond to the 271 form central charges. We next consider the 0-branes. We have a 10-component T-duality vector of fundamental 0-branes, a 16 component T-duality spinor of Dirichlet 0-branes, a singlet string soliton and a singlet KK monopole adding up to a total of 28 branes. The KK monopole correspond to the 10 -form central charge while the remaining 27 0-branes correspond to the $\mathbf{2 7} 0$-form central charge.

$\boldsymbol{D}=4$. Finally, we consider $D=4$ dimensions with 0 -branes only. We have a 12 component T-duality vector of fundamental 0-branes, a 32-component T-duality spinor of Dirichlet 0-branes and a 12-component T-duality vector of solitonic 0-branes adding up to a total of 56 branes. Sixteen of the Dirichlet 0-branes together with the 12 fundamental 0-branes correspond to the $\mathbf{2 8} 0$-form central charges while the remaining 16 Dirichlet 0-branes together with the 12 solitonic 0-branes correspond to the other $\mathbf{2 8} 0$-form central charges.

We conclude that the T-duality properties of the fundamental branes, Dirichlet branes, solitonic branes and KK monopoles precisely fit with the counting of the central charges in the supersymmetry algebra. This nicely confirms the results obtained in this paper. 


\section{$7 \quad \mathrm{E}_{11}$ and a ten-dimensional origin}

In section 2 we have derived the T-duality representations of all solitonic $p$-form potentials in $D<10$ dimensions by decomposing the known U-duality representations into T-duality representations. Remarkably, unlike D-branes, these $D<10$ T-duality representations cannot be obtained by a toroidal reduction of the $D=10$ solitonic $p$-form potentials. In other words, the lower dimensional $p$-form potentials do not possess a ten-dimensional origin in the context of the established IIA and IIB supergravity multiplets. One needs further $D=10$ representations to obtain the complete $D<10$ T-duality multiplets. One way to achieve this, is to assume that additional mixed-symmetry representations can be added to the ten-dimensional supergravity multiplets without upsetting the counting of degrees of freedom. It turns out that precisely those mixed-symmetry fields are needed that are predicted by the Kac-Moody algebra $\mathrm{E}_{11}$. With the present technology, it is not known how to extend the existing supergravity multiplets with mixed-symmetry fields at the level of the full non-linear supersymmetry algebra. This is only understood at the linearized level. Nevertheless, it is of interest to see that, once we assume that mixed-symmetry fields can be included, the $\mathrm{E}_{11}$ Kac-Moody algebra predicts precisely which fields are needed to re-obtain T-duality after toroidal compactification.

With the above motivation in mind we will show in this section how an $\mathrm{E}_{11}$ analysis can be used not only to derive all solitonic $p$-form potentials of IIA and IIB supergravity but also to derive all solitonic mixed symmetry fields that occur in the IIA and IIB $\mathrm{E}_{11}$ spectrum. In the first part of this section we will determine all solitonic fields, be it $p$ form potentials or mixed-symmetry fields, that arise in the $\mathrm{E}_{11}$ decomposition relevant for the IIA and the IIB theory. Once these fields have been determined, we will show in the second part of this section how, after a toroidal reduction, they give rise to precisely the same $D<10$ solitonic T-duality multiplets derived earlier in section 2 by decomposing in each dimension the U-duality representations of each $p$-form with respect to T-duality, with the result summarised in table $2 .^{7}$

\section{1 $\quad \mathrm{E}_{11}$ and mixed-symmetry fields}

In this subsection we will construct a procedure that selects out of the IIA and IIB $\mathrm{E}_{11}$ spectrum precisely those $p$-forms and mixed-symmetry fields that are solitonic, i.e. correspond to $\alpha=-2$. For the reader who wants to skip the technical derivation the final result, which coincides for the IIA and IIB theory, can be found in eq. (7.14).

Given the Dynkin diagram of $\mathrm{E}_{11}$ in figure 1, deleting any node in the horizontal line other than node 10 (in the nine-dimensional case one has to delete both nodes 9 and 11) results in a subgroup $\mathrm{GL}(D, \mathbb{R}) \times \mathrm{E}_{11-D}$, and the positive level generators ${ }^{8}$ with completely antisymmetric $\operatorname{GL}(D, \mathbb{R})$ indices are associated to the fields of the $D$ dimensional supergravity theory. In particular, in [7] this decomposition was obtained by explicitly performing a

\footnotetext{
${ }^{7}$ The strategy here is similar to the one in [7], where all the eleven-dimensional $\mathrm{E}_{11}$ fields giving rise to forms after dimensional reduction down to any dimension above two were determined.

${ }^{8}$ Given the decomposition of a positive root in terms of simple roots, the "level" associated to a given simple root is the number of times this simple root occurs.
} 


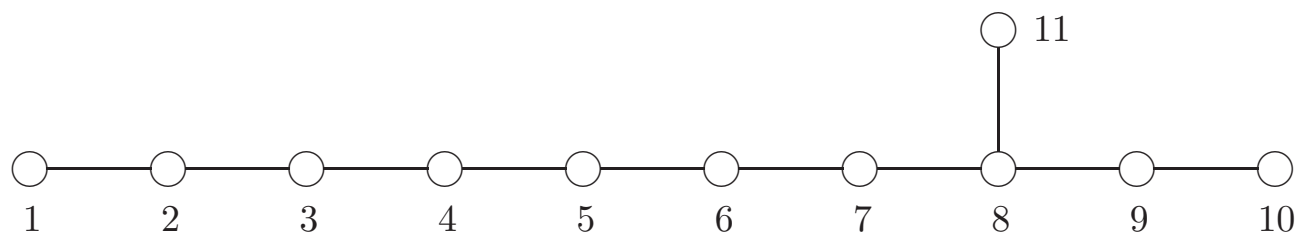

Figure 1. The $\mathrm{E}_{11}$ Dynkin diagram.

dimensional reduction of the generators associated to eleven dimensions, that is the positive level generators that are representations of the $G L(11, \mathbb{R})$ subgroup that results from deleting node 11. Most of these generators do not belong to completely antisymmetric representations, and therefore the corresponding fields are not forms. Nonetheless, some of these fields give rise to forms after dimensional reduction. Correspondingly, the supersymmetry algebra does not close on these fields in the eleven dimensional theory (it does only at the linearised level), but it does in the lower dimensional one as long as in the lower dimensional theory they are forms.

In [9] eleven dimensional supergravity was originally shown to be associated to the $\mathrm{E}_{11}$ algebra after a decomposition that results from deleting node 11, while its dimensional reduction to the IIA theory is obtained by considering the decomposition that results from the further deletion of node 10 [9]. The IIB theory was shown to result from $\mathrm{E}_{11}$ decomposed via the deletion of node 9 [29], while the full form field content of both IIA and IIB, including the 10-forms, was obtained in [30]. This was then shown [2-4] to be the field content on which the IIA and IIB supersymmetry algebras close.

In this paper we want to determine how the fields of lower-dimensional maximal supergravities form representations of the T-duality group, and we are thus interested in the dilaton weight of the ten-dimensional $\mathrm{E}_{11}$ fields. We consider the decomposition of the $\mathrm{E}_{11}$ algebra which is appropriate to either the IIB or the IIA theory. As already recalled, the IIB and the IIA generators correspond to decomposing the $\mathrm{E}_{11}$ algebra with respect to the two $\mathrm{A}_{9}$ subalgebras that arise from either deleting node 9 (IIB case) or nodes 10 and 11 (IIA case). In both cases, the way the fields scale with respect to the dilaton is dictated by the level associated to node 10, which we call $m$ [11]. This means that in the corresponding positive root of $\mathrm{E}_{11}$ the simple root $\alpha_{10}$ occurs $m$ times. The relation between $m$ and $\alpha$ is simply $[11]^{9}$

$$
m=-\alpha .
$$

We use now the strategy of refs. [31-33] to decompose the roots of $\mathrm{E}_{11}$ level by level in terms of representations of a finite dimensional subalgebra. We first concentrate on the IIB case. The decomposition of the $\mathrm{E}_{11}$ roots which is appropriate to IIB was performed in [34], and here we review these results. We denote with $\alpha_{i}$ the simple roots associated to nodes 1-8 and node 11. These are the simple roots of the $\mathrm{A}_{9}$ of IIB. Given a positive root

\footnotetext{
${ }^{9}$ The parameter $\alpha$ should not be confused with the $\mathrm{E}_{11}$ roots, which are also denoted by $\alpha$.
} 
$\alpha$ of $\mathrm{E}_{11}$, one can then write

$$
\alpha=\sum_{i} n_{i} \alpha_{i}+l \alpha_{9}+m \alpha_{10}
$$

where $l$ and $m$ are the levels associated to nodes 9 and 10. One then writes the simple root $\alpha_{9}$ as

$$
\alpha_{9}=x-\frac{1}{2} \alpha_{10}-\lambda_{8},
$$

where $x$ is a vector in the direction orthogonal to the simple roots $\alpha_{i}$ and $\alpha_{10}$, and where $\lambda_{8}$ is the fundamental weight of the $A_{9}$ subalgebra associated to node 8 , that is

$$
\lambda_{8}=\frac{1}{5}\left[\alpha_{1}+2 \alpha_{2}+3 \alpha_{3}+\ldots+8 \alpha_{8}+4 \alpha_{11}\right]
$$

and

$$
\lambda_{8}^{2}=\frac{8}{5} .
$$

This gives

$$
x^{2}=-\frac{1}{10},
$$

given that all the simple roots have square length 2 . In order to have a representation of $\mathrm{A}_{9}$ denoted by the Dynkin indices $p_{i}$ in the adjoint of $\mathrm{E}_{11}$, a necessary condition is that the highest weight $\sum_{i} p_{i} \lambda_{i}$ occurs when one projects the positive roots along the space of the simple roots $\alpha_{i}$ of $\mathrm{A}_{9}$. This means that one must have

$$
\alpha=\sum_{i} p_{i} \lambda_{i}+l x+\left(m-\frac{l}{2}\right) \alpha_{10} .
$$

As shown in [34], there is a relation between the level $l$ and the number of GL(10, R) indices of the corresponding generators. This relation is dictated by the fact that the root $\alpha_{9}$ corresponds to a generator with 2 antisymmetric indices, and thus at level $l$ one finds that all generators must have $2 l$ indices. Including also the possibility that there are groups of 10 antisymmetric indices, one obtains the condition

$$
10 n+\sum_{i} p_{i}(10-i)=2 l,
$$

where $n$ is the number of groups of 10 antisymmetric indices. Another constraint comes from imposing that the roots must have square length at most 2 , or more precisely $\alpha^{2}=$ $2,0,-2, \ldots[35]$. Using eq. (7.6) one gets ${ }^{10}$

$$
\alpha^{2}=\sum_{i j} p_{i} A_{i j}^{-1} p_{j}+F_{B}(l, m)
$$

where

$$
F_{B}(l, m)=\frac{2}{5} l^{2}+2 m^{2}-2 m l
$$

\footnotetext{
${ }^{10}$ This formula is identical to eq. (27) of ref. [34], as can be seen using eq. (26) in that paper, which relates the level $m$ to the $\mathrm{SL}(2, \mathbb{R})$ weight $q$.
} 
and where $A_{i j}^{-1}$ is the inverse of the $\mathrm{A}_{9}$ Cartan matrix,

$$
A_{i j}^{-1}=\left(\lambda_{i}, \lambda_{j}\right)
$$

For the general $\mathrm{A}_{n-1}$ algebra the inverse matrix is given by

$$
\left(A_{j k}\right)^{-1}=\left\{\begin{array}{ll}
\frac{j(n-k)}{n}, & j \leq k \\
\frac{k(n-j)}{n}, & j \geq k
\end{array} .\right.
$$

The strategy is now to analyse all possible representations at levels $l$ and $m$ that satisfy the condition of having $2 l$ indices and such that $\alpha^{2}$ has one of the allowed values $2,0,-2, \ldots$ This analysis does not give information about the actual multiplicity of the representations, which one can obtain by comparison with listed results (see for instance the tables in ref. [30]). One can immediately see by direct inspection that for $m=0$ one can only get a solution for $l=0$ and $l=1$. In particular, the generator corresponding to the $l=1$ solution is an object with 2 antisymmetric indices, and the corresponding field is the fundamental 2-form $B_{2}$. One has

$$
F_{B}(l, 0)>2 \text { for } l>2
$$

and therefore there are no solutions for $l>2$. Also the case $l=2$ has no solution.

For $m=1$ one has solutions for $l=0,1, \ldots, 5$, the corresponding fields have completely antisymmetric indices and are the RR fields $C_{2 l}$ of the IIB theory. It is quite easy to see that there are no solutions for $l \geq 6$. Therefore we have shown that the only fields at level $m=1$ are the IIB RR forms. We will see that the same result applies to the IIA theory. This is all in agreement with the results of [10], which show that all the RR forms in lower dimension are obtained from dimensional reduction of the ten-dimensional ones.

Similarly, one can consider $m=2$. In this case it is easy to see by direct substitution that there are no solutions if $l=0, l=1$ and $l=2$, as well as for $l \geq 8$. The only solutions are

$$
\begin{aligned}
& l=3: A_{6} \\
& l=4: A_{7,1} \quad A_{8} \\
& l=5: A_{8,2} \quad A_{9,1} \quad 2 \times A_{10} \\
& l=6: A_{9,3} \quad A_{10,2} \\
& l=7: A_{10,4},
\end{aligned}
$$

where the multiplicity 2 of the 10 -form $A_{10}$ is read from [30]. The above result summarizes all solitonic $p$-form potentials and mixed symmetry fields contained in the IIB decomposition of $\mathrm{E}_{11}$.

We now consider the IIA case. We can obtain the IIA theory by first deleting node 11 and then further decomposing by deleting node 10, or the other way around. We denote with $l$ the level associated with the simple root $\alpha_{11}$, and with $m$ the level associated with the simple root $\alpha_{10}$, while the roots from 1 to 9 are denoted by $\alpha_{i}$ (we deliberately use the 
same notation as in the IIB case, although the decomposition of the algebra is different). We write a generic positive root as

$$
\alpha=\sum_{i} n_{i} \alpha_{i}+l \alpha_{11}+m \alpha_{10}
$$

We first delete node 11, and we write

$$
\alpha_{11}=y-\mu_{8}
$$

where the vector $y$ is orthogonal to the $\mathrm{A}_{10}$ space of simple roots $\alpha_{1}, \ldots \alpha_{10}$, and $\mu_{8}$ is the fundamental weight associated to node 8 in $\mathrm{A}_{10}$, that is

$$
\mu_{8}=\frac{3}{11}\left[\alpha_{1}+2 \alpha_{2}+\ldots+8 \alpha_{8}+\frac{16}{3} \alpha_{9}+\frac{8}{3} \alpha_{10}\right]
$$

and

$$
\mu_{8}^{2}=\frac{24}{11}
$$

This gives

$$
y^{2}=-\frac{2}{11} .
$$

Once (7.16) is plugged into (7.15), one can see that the coefficient of the root $\alpha_{10}$ is $m-\frac{8}{11} l$. If we now delete node 10 , we write the simple root $\alpha_{10}$ as

$$
\alpha_{10}=z-\lambda_{9},
$$

where $z$ is a vector orthogonal to the simple roots of $A_{9}$ (and orthogonal to $y$ as well), and where $\lambda_{9}$ is the fundamental weight of $\mathrm{A}_{9}$ associated to node 9 , that is

$$
\lambda_{9}=\frac{1}{10}\left[\alpha_{1}+2 \alpha_{2}+\ldots+9 \alpha_{9}\right]
$$

and

$$
\lambda_{9}^{2}=\frac{9}{10},
$$

which gives

$$
z^{2}=\frac{11}{10} .
$$

Repeating the same argument as for IIB, in order to have a representation of highest weight state $\sum_{i} p_{i} \lambda_{i}$, we must have

$$
\alpha=\sum_{i} p_{i} \lambda_{i}+l y+\left(m-\frac{8}{11} l\right) z .
$$

Using (7.19) and (7.23) one gets

$$
\alpha^{2}=\sum_{i j} p_{i} A_{i j}^{-1} p_{j}+F_{A}(l, m),
$$


where

$$
F_{A}(l, m)=\frac{2}{5} l^{2}+\frac{11}{10} m^{2}-\frac{8}{5} m l .
$$

One gets exactly the same result if one first deletes node 10 and then node 11 . In this case one writes

$$
\alpha_{10}=\tilde{z}-\tilde{\mu}_{9},
$$

where now $\tilde{z}$ is orthogonal to the space of simple roots $\alpha_{1}, \ldots, \alpha_{9}$ and $\alpha_{11}$ which form the algebra $\mathrm{D}_{10}$, and $\tilde{\mu}_{9}$ is the $\mathrm{D}_{10}$ fundamental weight associated to node 9 ,

$$
\tilde{\mu}_{9}=\frac{1}{2}\left[\alpha_{1}+2 \alpha_{2}+\ldots+8 \alpha_{8}+5 \alpha_{9}+4 \alpha_{11}\right] .
$$

This gives

$$
\tilde{\mu}_{9}^{2}=\frac{5}{2}
$$

which implies

$$
\tilde{z}^{2}=-\frac{1}{2}
$$

Once eq. (7.27) is substituted in eq. (7.16), one can see that the coefficient in front of the root $\alpha_{11}$ is $(l-2 m)$. If we now delete node 11 , this corresponds to writing

$$
\alpha_{11}=\tilde{y}-\lambda_{8},
$$

where $\tilde{y}$ is orthogonal to the $A_{9} \operatorname{roots} \alpha_{i}$ and to $\tilde{z}$, and $\lambda_{8}$ is

$$
\lambda_{8}=\frac{1}{5}\left[\alpha_{1}+2 \alpha_{2}+3 \alpha_{3}+\ldots+8 \alpha_{8}+4 \alpha_{9}\right],
$$

which implies

$$
\lambda_{8}^{2}=\frac{8}{5}
$$

and

$$
\tilde{y}^{2}=\frac{2}{5}
$$

Using these results one obtains again the expression (7.25) for the square length of the root.

We now want to analyse the representations that arise at each level. Again, we must impose $\alpha^{2}=2,0,-2 \ldots$ We also have a constraint on the $\mathrm{GL}(10, \mathbb{R})$ representations coming from imposing that the number of indices of a generator at levels $(l, m)$ must be $2 l+m$, that is

$$
10 n+\sum_{i} p_{i}(10-i)=2 l+m
$$

where again $n$ is the number of groups of 10 antisymmetric indices. For $m=0$ we get the same result as in IIB, while for $m=1$ we only get solutions for $l=0,1, \ldots, 4$, the corresponding fields being the RR forms, $C_{2 l+1}$.

It is important to emphasise that by simply looking at eqs. (7.10) and (7.26) one deduces that for any given $m$ there is a finite number of solutions, that is a finite number of representations, in both IIA and IIB. A more careful analysis reveals that

$$
F_{B}\left(l+\frac{m}{2}, m\right)=F_{A}(l, m) .
$$


This remarkable result implies that when $m$ is even the analysis of the representations for IIB and IIA is identical, that is for any given $m$ the representations that one gets at a given level $l$ for IIA are the same as the ones that one gets at level $l+\frac{m}{2}$ for IIB. If one considers all the representations with a given even $m$, the IIA and IIB thus give the same result. In particular, this implies that the $m=2$ fields are the same for both theories, and they are given in (7.14). Given that the level must be integer, nothing can be said when $m$ is odd, and there is no way to compare the representations of IIA with the representations of IIB in this case.

\section{2 $\quad \mathrm{E}_{11}$ and T-duality}

In the previous subsection we have determined all solitonic fields in $D=10$ dimensions, $p$-forms and mixed-symmetry fields included, and found the following result for both IIA and IIB:

$$
A_{6} \quad A_{7,1} \quad A_{8} \quad A_{8,2} \quad A_{9,1} \quad A_{9,3} \quad 2 \times A_{10} \quad A_{10,2} \quad A_{10,4} .
$$

We now proceed with a reduction of all these fields on a $d$-torus, where $d=10-D$, and we only keep the resulting $p$-forms, that is we only consider the $D$-dimensional spacetime indices to be completely antisymmetric. Each field, when reduced, is decomposed in forms that are representations of $\operatorname{SL}(d, \mathbb{R})$, and we will show that summing for each form the representations of $\mathrm{SL}(d, \mathbb{R})$ resulting from all the 10-dimensional fields we obtain representations of the T-duality group $\mathrm{SO}(d, d)$.

We consider $D \geq 3$, while the cases $D<3$ will be considered separately at the end of this section. The lowest form that one can get is a $D-4$-form (this form only exists for $D \geq 4$ ), which corresponds to $A_{6}$ with the highest possible amount of internal indices:

$$
A_{D-4, i_{1} \ldots i_{d}} \rightarrow \mathbf{1}
$$

This is a singlet of $\mathrm{SL}(d, \mathbb{R})$, which is of course also a singlet of $\mathrm{SO}(d, d)$.

The $D-3$ forms come from $A_{6}$,

$$
A_{D-3, i_{1} \ldots i_{d-1}} \rightarrow \overline{\mathbf{d}}
$$

and from $A_{7,1}$,

$$
A_{D-3, i_{1} \ldots i_{d}, j} \rightarrow \mathbf{d} .
$$

This gives the vector representation of $\mathrm{SO}(d, d)$ as results from

$$
\mathbf{2 d}=\mathbf{d} \oplus \overline{\mathbf{d}} .
$$

Next we consider the $D-2$-forms. They come from $A_{6}$,

$$
A_{D-2, i_{1} \ldots i_{d-2}} \rightarrow(\overline{\mathbf{d}} \otimes \overline{\mathbf{d}})_{A},
$$

from $A_{7,1}$,

$$
A_{D-2, i_{1} \ldots i_{d-1}, j} \rightarrow \mathbf{d} \otimes \overline{\mathbf{d}}
$$


from $A_{8}$,

$$
A_{D-2, i_{1} \ldots i_{d}} \rightarrow \mathbf{1}
$$

and from $A_{8,2}$,

$$
A_{D-2, i_{1} i_{2}} \rightarrow(\mathbf{d} \otimes \mathbf{d})_{A} .
$$

Here and in the rest of this section we denote with the suffix $A$ the antisymmetrised product of the representations. Summing up all the representations of the $D-2$-forms gives the $\mathrm{SO}(d, d)$ representations $\mathbf{1} \oplus(\mathbf{2} \mathbf{d} \otimes \mathbf{2 d})_{A}$, where

$$
(\mathbf{2} \mathbf{d} \otimes \mathbf{2} \mathbf{d})_{A}=(\mathbf{d} \otimes \mathbf{d})_{A} \oplus(\mathbf{d} \otimes \overline{\mathbf{d}}) \oplus(\overline{\mathbf{d}} \otimes \overline{\mathbf{d}})_{A} .
$$

We then consider the $D-1$-forms. From $A_{6}$ we get

$$
A_{D-1, i_{1} \ldots i_{d-3}} \rightarrow(\overline{\mathbf{d}} \otimes \overline{\mathbf{d}} \otimes \overline{\mathbf{d}})_{A},
$$

from $A_{7,1}$ we get

$$
A_{D-1, i_{1} \ldots i_{d-2}, j} \rightarrow \mathbf{d} \otimes(\overline{\mathbf{d}} \otimes \overline{\mathbf{d}})_{A}
$$

from $A_{8}$ we get

$$
A_{D-1, i_{1} \ldots i_{d-1}} \rightarrow \overline{\mathbf{d}}
$$

from $A_{8,2}$ we get

$$
A_{D-1, i_{1} \ldots i_{d-1}, j_{1} j_{2}} \rightarrow \overline{\mathbf{d}} \otimes(\mathbf{d} \otimes \mathbf{d})_{A}
$$

from $A_{9,1}$ we get

$$
A_{D-1, i_{1} \ldots i_{d}, j} \rightarrow \mathbf{d}
$$

and finally from $A_{9,3}$ we get

$$
A_{D-1, i_{1} \ldots i_{d}, j_{1} j_{2} j_{3}} \rightarrow(\mathbf{d} \otimes \mathbf{d} \otimes \mathbf{d})_{A} .
$$

This gives $\mathbf{2} \mathbf{d} \oplus(\mathbf{2} \mathbf{d} \otimes \mathbf{2 d} \otimes \mathbf{2} \mathbf{d})_{A}$ of $\mathrm{SO}(d, d)$, where

$$
(\mathbf{2 d} \otimes \mathbf{2 d} \otimes \mathbf{2} \mathbf{d})_{A}=(\mathbf{d} \otimes \mathbf{d} \otimes \mathbf{d})_{A} \oplus\left[\overline{\mathbf{d}} \otimes(\mathbf{d} \otimes \mathbf{d})_{A}\right] \oplus\left[\mathbf{d} \otimes(\overline{\mathbf{d}} \otimes \overline{\mathbf{d}})_{A}\right] \oplus(\overline{\mathbf{d}} \otimes \overline{\mathbf{d}} \otimes \overline{\mathbf{d}})_{A} .
$$

Finally, we consider the $D$-forms. All the fields in eq. (7.37) contribute, and we list here the $\operatorname{SL}(d, \mathbb{R})$ representations for all the fields, in the same order as they appear in eq. (7.37):

$$
\begin{aligned}
& (\overline{\mathbf{d}} \otimes \overline{\mathbf{d}} \otimes \overline{\mathbf{d}} \otimes \overline{\mathbf{d}})_{A} \\
& \mathbf{d} \otimes(\overline{\mathbf{d}} \otimes \overline{\mathbf{d}} \otimes \overline{\mathbf{d}})_{A} \\
& (\overline{\mathbf{d}} \otimes \overline{\mathbf{d}})_{A} \\
& (\mathbf{d} \otimes \mathbf{d})_{A} \otimes(\overline{\mathbf{d}} \otimes \overline{\mathbf{d}})_{A} \\
& \mathbf{d} \otimes \overline{\mathbf{d}} \\
& \overline{\mathbf{d}} \otimes(\mathbf{d} \otimes \mathbf{d} \otimes \mathbf{d})_{A} \\
& 2 \times \mathbf{1} \\
& (\mathbf{d} \otimes \mathbf{d})_{A} \\
& (\mathbf{d} \otimes \mathbf{d} \otimes \mathbf{d} \otimes \mathbf{d})_{A} .
\end{aligned}
$$


This sums up to $\mathbf{1} \oplus \mathbf{1} \oplus(\mathbf{2} \mathbf{d} \otimes \mathbf{2 d})_{A} \oplus(\mathbf{2} \mathbf{d} \otimes \mathbf{2} \mathbf{d} \otimes \mathbf{2} \mathbf{d} \otimes \mathbf{2 d})_{A}$ of $\mathrm{SO}(d, d)$. In particular, the representation corresponding to four antisymmetrised $\mathrm{SO}(d, d)$ indices decomposes as

$$
\begin{aligned}
& (\mathbf{2} \mathbf{d} \otimes \mathbf{2} \mathbf{d} \otimes \mathbf{2} \mathbf{d} \otimes \mathbf{2 d})_{A}=(\mathbf{d} \otimes \mathbf{d} \otimes \mathbf{d} \otimes \mathbf{d})_{A} \oplus\left[\overline{\mathbf{d}} \otimes(\mathbf{d} \otimes \mathbf{d} \otimes \mathbf{d})_{A}\right] \\
& \oplus\left[(\mathbf{d} \otimes \mathbf{d})_{A} \otimes(\overline{\mathbf{d}} \otimes \overline{\mathbf{d}})_{A}\right] \oplus\left[\mathbf{d} \otimes(\overline{\mathbf{d}} \otimes \overline{\mathbf{d}} \otimes \overline{\mathbf{d}})_{A}\right] \oplus(\overline{\mathbf{d}} \otimes \overline{\mathbf{d}} \otimes \overline{\mathbf{d}} \otimes \overline{\mathbf{d}})_{A}
\end{aligned}
$$

Summarizing, we find that the complete result for the solitonic $p$-form potentials precisely coincides with the one of section 2 which is summarised in table 2. Besides, this general analysis also shows in a very elegant and concise way that the same set of solitonic fields occurs in four and three dimensions, as anticipated in table 2.

To complete the analysis, we also derive the forms that arise from the reduction of the fields in eq. (7.37) to $D<3$. We first consider the case $D=2$. In the derivation of the scalars, that is the 0 -forms, with respect to the general derivation of the $D-2$ forms in higher dimensions given in eqs. (7.42)-(7.45), it is eq. (7.43) that gets modified. Indeed, given that all the indices of the field $A_{7,1}$ are internal, we have to use the fact that the $\operatorname{GL}(10, \mathbb{R})$ representation of the field is irreducible, which results in the $\mathbf{6 3}$ of $\mathrm{SL}(8, \mathbb{R})$ after reduction, while eq. (7.43) applied to the $D=2$ case would have given an additional singlet. All the other representations in $D=2$ are unchanged. To summarise, the $D$ fields in two dimensions are

$$
D_{0, A B} \quad D_{1, A} \quad D_{1, A B C} \quad D_{2} \quad D_{2}^{\prime} \quad D_{2, A B} \quad D_{2, A B C D} .
$$

Repeating the same argument, for $D=1$ one finds

$$
D_{0, A B C} \quad D_{1} \quad D_{1, A B} \quad D_{1, A B C D} .
$$

One can formally also reduce to zero dimensions, which gives

$$
D_{0} \quad D_{0, A B C D}
$$

This last result, not surprisingly, is what one would get by decomposing the adjoint representation of $E_{11}$ in terms of the $D_{10}$ algebra which results from deleting node 10 in the Dynkin diagram of figure 1 and reading the result at level 2.

\section{Conclusions}

In this paper we extended our previous work on the T-duality covariant formulation of WZ terms corresponding to Fundamental Branes and D-Branes to include the WZ terms of String Solitons, see eq. (5.8). This led to two distinguishing features which did not occur in the analysis of the Fundamental Branes and D-branes. First of all, not all solitonic potentials of supergravity correspond to a supersymmetric String Soliton. This is due to the fact that the construction of a gauge-invariant WZ term requires the introduction of a number of worldvolume potentials that do not always fit into a worldvolume multiplet with 16 supercharges. First, we have determined the T-duality multiplets which contain the String Solitons, see table 1. Next, we identified the conjugacy classes within these 
T-duality multiplets to which the String Solitons belong. These conjugacy classes can be defined by specifying a certain set of lightlike directions, see eq. (5.9). Their dimensions have been determined, see table 9 .

The second distinguishing feature is that the supersymmetric String Solitons fill out T-duality conjugacy classes, whose ten-dimensional origin cannot be understood by considering the standard NS5A and NS5B branes of string theory alone. The missing components can be understood as arising from the reduction of a number of mixed symmetry fields that are precisely the ones predicted by the very extended Kac-Moody algebra $\mathrm{E}_{11}$. This is only formally so since the mixed symmetry fields, with the present technology, can only be defined for linearised supersymmetry.

A special example of a mixed symmetry solitonic potential, predicted by $\mathrm{E}_{11}$, is the dual graviton [9]. ${ }^{11}$ In $D=10$ dimensions this is a mixed symmetry potential $A_{7,1}$ which has 8 indices but is only antisymmetric in the first 7 indices. Although this dual graviton can only be defined for linearised gravity and linearised supersymmetry [37], upon reduction to 9 dimensions it gives rise to a solitonic 6 -form and 7 -form potential that are part of the non-linear 9-dimensional supergravity theory. These potentials are the duals of the KaluzaKlein $(\mathrm{KK})$ vector and KK scalar, respectively. In particular, the 5-brane charged under the 6 -form is the reduction in the isometry direction of the KK5A (KK5B) monopole, whose worldvolume theory is described by a vector (tensor) multiplet [28]. This brane, together with the unwrapped NS5A (NS5B) forms a vector of the nine-dimensional T-duality group $\mathrm{SO}(1,1)$, which splits in selfdual and anti-selfdual representations. In lower dimensions the picture is analogous. In particular in $D$ dimensions the KK monopole, reduced in the isometry direction and wrapped on a $d$-1-dimensional torus, and the NS5-brane, reduced in a transverse direction and wrapped on a $d$-1-dimensional torus, give $D$-4-branes that transform as vectors of $\mathrm{SO}(d, d)$. The important point is that, in order to obtain the full T-duality vector representation one needs to include the dual graviton. ${ }^{12}$ The same applies to all the other mixed-symmetry potentials predicted by $\mathrm{E}_{11}$. They are needed to understand the 10-dimensional origin of the different soliton conjugacy classes.

It is interesting to compare the relation between branes and doubled geometry in more detail. For the Fundamental Branes and the D-branes we found that the WZ term of the Fundamental 0-branes (see eq. (1.6)), does not contain extra scalars, i.e. they are insensitive for the doubled geometry. In contrast, the WZ term of the Fundamental String (see eq. (1.5)) and the D-branes (see eq. (1.8)) depends on twice as many extra scalars as there are compactified dimensions, i.e. the Fundamental String and the D-branes feel the doubled geometry. For the Solitonic Branes the situation is slightly different. These branes depend on the extra scalars via the world-volume curvature $\mathcal{F}_{1, A}$ with the index $A$ uncontracted. In

\footnotetext{
${ }^{11}$ Another nice application of the mixed symmetry potentials, predicted by $\mathrm{E}_{11}$, is the understanding, be it at the linearized level only, of the 11-dimensional origin of the IIA 9-form RR potential that is dual to Romans mass parameter $m$ [36]. This is achieved by the mixed symmetry potential $A_{10,1,1}$, with 10 antisymmetric indices and 2 symmetric ones.

${ }^{12}$ The dual graviton is special in the sense that there is a corresponding KK monopole solution which can be described in terms of the metric. Similar solutions do not exist for the other mixed symmetry fields that are needed to fill out the T-duality multiplets.
} 
section 5 we have seen that this implies that a particular Solitonic Brane, which transforms as an anti-symmetric tensor with $m$ indices under T-duality, only depends on $m$ out of the $2 d$ extra scalars. The doubled geometry, or T-duality covariance, implies that the Tdual rotated Solitonic Brane will sense the T-dual rotated extra scalars, but again this rotated brane will only depend on $m$ out of the $2 d$ extra scalars. The fact that there is no need to impose duality relations on the extra scalars, like in the case of the Fundamental String [19-21], should make the construction of a kappa-symmetric action easier.

The String Solitons, with brane tension $T \sim\left(g_{s}\right)^{-2}$, are just one step in a whole family of interconnected branes with tension $T \sim\left(g_{s}\right)^{\alpha}$ and $\alpha=0,-1,-2, \cdots$ etc. The next set of branes in this family, corresponding to $\alpha=-3$, are much harder to understand. There is a crucial difference between the fields with $\alpha \leq-3$ and the ones with $\alpha=0,-1$ and -2 at the level of the gauge algebra. The solitonic fields, given in table 2 , have the same structure in any dimension, and given that their transformations contain RR fields in the form of $\mathrm{SO}(d, d)$ spinor bilinears, the whole analysis of the gauge algebra can be performed in a general way which is the same in any dimension, as was shown in section 4 . For the $\alpha=-3$ fields the situation is different, because the requirement of gauge invariance involves the cancellation of terms containing three $\alpha=-1$ objects. This is achieved in each dimension using Fierz identities of spinors of $\mathrm{SO}(d, d)$, which implies that in this case the analysis is dimension-dependent.

An example of an $\alpha=-3$ field is the field $E_{8}$ of IIB, which is the S-dual of the RR 8-form $C_{8}$. Its field strength and gauge transformations are

$$
\begin{aligned}
K_{9} & =d E_{8}+G_{3} D_{6}-\frac{1}{2} F_{7} C_{2} \\
\delta E_{8} & =d \Xi_{7}+G_{3} \Lambda_{5}-\frac{1}{2} F_{7} \lambda_{1},
\end{aligned}
$$

and one can easily write down a corresponding WZ term, which contains the world volume fields $c_{1}$ and $d_{5}$ together with two embedding scalars. Imposing electromagnetic duality between $c_{1}$ and $d_{5}$ one obtains a vector plus two scalars, which is the bosonic sector of a vector multiplet on an 8-dimensional world volume, and the corresponding brane is the S-dual of the D7-brane. As can be seen from tables $3-7$, the $\alpha=-3$ field of lowest rank is always $E_{D-2, \dot{a}}$, and one can show that this field always gives a WZ term corresponding to a supersymmetric brane. This brane belongs to the anti-chiral spinor $\mathrm{SO}(d, d)$ representation, which contains the double dimensional reduction of the S-dual of the D7-brane of IIB. One can also perform direct dimensional reductions of this object, and correspondingly one expects to find branes associated to $\alpha=-3$ fields of higher rank. All these branes can be seen as the endpoints of Fundamental Branes, D-branes and String Solitons, and it would be interesting to determine their T-duality representations and to investigate them in more detail.

We just saw an example of a supersymmetric $\alpha=-3$ brane which was the S-dual of a Dirichlet brane. By performing similar U-duality rotations on the other branes one can easily construct examples of supersymmetric branes with $\alpha \leq-3$. The minimum values of $\alpha$ one can obtain in this way are indicated in table 11 . The cases $D=3$ and $D=4$, where the lowest values of $\alpha$ are obtained, are a bit special. While in dimensions higher than four 


\begin{tabular}{|c|c|c|c|c|c|c|c|c|c|}
\hline $\mathrm{D}$ & IIA & IIB & 9 & 8 & 7 & 6 & 5 & 4 & 3 \\
\hline$\alpha_{\min }$ & -2 & -4 & -4 & -4 & -4 & -5 & -5 & -7 & -11 \\
\hline
\end{tabular}

Table 11. This table gives the minimum value $\alpha_{\min }$ corresponding to the potentials of maximal supergravity in $D$ dimensions.

the U-duality group $\mathrm{E}_{d+1(d+1)}$ decomposes as

$$
\mathrm{E}_{d+1(d+1)} \supset \mathrm{SO}(d, d) \times \mathbb{R}^{+}
$$

in four dimensions one has

$$
\mathrm{E}_{7(7)} \supset \mathrm{SO}(6,6) \times \mathrm{SL}(2, \mathbb{R})
$$

and in three dimensions one has

$$
\mathrm{E}_{8(8)} \supset \mathrm{SO}(8,8)
$$

This implies that in order to obtain tables equivalent to tables $3-7$, and in particular in order to determine the dilaton weight of the fields, one has to perform a further decomposition. The U-duality representations of all the forms in $D=4$ and $D=3$ are given in $[7,8]$. In four dimensions the $\mathrm{SL}(2, \mathbb{R})$ symmetry implies that for each $p$-form, the T-duality representation corresponding to $\alpha$ and the one corresponding to $-\alpha-2 p$ are the same, and the lowest value of $\alpha$, corresponding to a 4 -form, is -7 , as can be deduced from the fact that the highest value of $\alpha$ for a 4 -form is -1 . In three dimensions, for each $p$-form the T-duality representation corresponding to $\alpha$ and the one corresponding to $-\alpha-4 p$ are conjugate, and the lowest value of $\alpha$, corresponding to a 3 -form, is -11 .

The fact that the D7-brane, with $\alpha=-1$, and the S-dual of the D7-brane, with $\alpha=-3$, are related to each other under U-duality, implies that the worldvolume dynamics of both branes is described by the same supermultiplet, which in this case is a vector multiplet. In view of this it is convenient to classify branes according to the supermultiplet that governs their worldvolume dynamics. Since we have only three different multiplets (scalar, vector and (self-dual) tensor) we only have three different kind of branes: Scalar, Vector and Tensor Branes. Using this terminology, the Fundamental Branes are Scalar Branes, while the Dirichlet brane are Vector Branes. ${ }^{13}$ Among the String Solitons we have Vector and Tensor Branes. All Vector Solitons can be obtained by a U-duality rotation of a Vector Dirichlet Brane. Note, however, that their T-duality representations are not the same. In this sense Vector Solitons are not truly independent branes. This is different from the Tensor Solitons which stand on themselves and cannot be obtained by a U-duality rotation of a Scalar or Vector brane.

To conclude, there are a huge number of branes in string theory. In this paper we have concentrated on just three classes of them: the Fundamental Branes, D-branes and Solitons. These are by far the best understood branes. Table 11 shows that there are many

\footnotetext{
${ }^{13}$ This terminology is not unique for low-dimensional worldvolumes since for three-dimensional worldvolumes a vector is dual to a scalar and for two-dimensional worldvolumes a vector is dual to an integration constant.
} 
other non-perturbative branes, whose present status is unclear. It would be interesting to bring some order in them and to investigate whether there is some role to play by these suggestive branes.

\section{Acknowledgments}

E.B. wishes to thank King's College London and F.R. wishes to thank the University of Groningen for hospitality. The work of F.R. was supported by the STFC rolling grant ST/G000/395/1.

\section{A Spinor representations of $\mathrm{SO}(d, d)$}

In this appendix we summarise a few useful properties of the spinors of $\mathrm{SO}(d, d)$, where $d=10-D$. We will partly follow [38], (appendix of [39]), although we will be using a different basis for the gamma matrices. Dirac spinors of $\mathrm{SO}(d, d)$ have $2^{d}$ components. Introducing gamma matrices $\Gamma_{A}(A=1 \cdots, 2 d)$, satisfying the Clifford algebra

$$
\left\{\Gamma_{A}, \Gamma_{B}\right\}=2 \eta_{A B}
$$

where $\eta_{A B}$ is the Minkowski metric with signature $(d, d)$, one defines the (unitary) charge conjugation matrix $C$, whose symmetry property is

$$
C^{T}=-\epsilon C
$$

where $\epsilon= \pm 1$, such that

$$
C \Gamma_{A} C^{\dagger}=-\eta \Gamma_{A}^{T},
$$

where $\eta= \pm 1$. The matrix $C$ is thus symmetric if $\epsilon=-1$ and antisymmetric if $\epsilon=1$, and the symmetry property of $C \Gamma_{A}$ is determined by the product of the two parameters $\epsilon$ and $\eta$,

$$
\left(C \Gamma_{A}\right)^{T}=\epsilon \eta\left(C \Gamma_{A}\right) .
$$

This enables one to calculate the symmetry property of any matrix $C \Gamma_{A_{1} \cdots A_{n}}$. Requiring that the total number of symmetric and antisymmetric matrices equals $\frac{1}{2} 2^{d}\left(2^{d}+1\right)$ and $\frac{1}{2} 2^{d}\left(2^{d}-1\right)$, respectively, one finds, for each value of $d$, two solutions for the pair $\epsilon, \eta$ such that eqs. (A.2) and (A.3) are satisfied. These solutions are listed in table 12.

Chiral spinors can be defined by introducing the matrix $\Gamma_{\star}$, with $\Gamma_{\star} \Gamma_{\star}=1$, which is proportional to the product of all other gamma-matrices:

$$
\Gamma_{\star}=(-)^{d} \Gamma_{1} \cdots \Gamma_{2 d}
$$

For all values of $D$ one can define Majorana-Weyl spinors with $2^{d-1}$ real components. In all dimensions we are using a Weyl basis, so that a spinor $\lambda_{\alpha}$ decomposes according to

$$
\lambda_{\alpha}=\left(\begin{array}{c}
\lambda_{a} \\
\lambda_{\dot{a}}
\end{array}\right),
$$




\begin{tabular}{|l|c|c|c|c|c|}
\hline$d$ & $\epsilon$ & $\eta$ & $C$ & $C \Gamma_{A}$ & {$\left[C, \Gamma_{\star}\right\}$} \\
\hline 1,5 & +1 & +1 & $\mathrm{~A}$ & $\mathrm{~S}$ & $\mathrm{AC}$ \\
& -1 & -1 & $\mathrm{~S}$ & $\mathrm{~S}$ & \\
\hline 2,6 & +1 & -1 & $\mathrm{~A}$ & $\mathrm{~A}$ & $\mathrm{C}$ \\
& +1 & +1 & $\mathrm{~A}$ & $\mathrm{~S}$ & \\
\hline 3,7 & -1 & +1 & $\mathrm{~S}$ & $\mathrm{~A}$ & $\mathrm{AC}$ \\
& +1 & -1 & $\mathrm{~A}$ & $\mathrm{~A}$ & \\
\hline 4,8 & -1 & -1 & $\mathrm{~S}$ & $\mathrm{~S}$ & $\mathrm{C}$ \\
& -1 & +1 & $\mathrm{~S}$ & $\mathrm{~A}$ & \\
\hline
\end{tabular}

Table 12. The possible values of $\epsilon$ and $\eta$ for various $d$. The symmetry properties of $C$ and $C \Gamma_{A}$ are indicated with S (symmetric) and A (anti-symmetric). The sixth column indicates whether the charge conjugation matrix commutes $(\mathrm{C})$ or anti-commutes $(\mathrm{AC})$ with $\Gamma_{\star}$. In each dimension, the charge conjugation matrix corresponding to the first line is $C_{1}$ and the one corresponding to the second line is $C_{2}$.

where the Dirac spinor index $\alpha, \alpha=1, \ldots, 2^{d}$ splits in the two indices $a$ and $\dot{a}$ denoting spinors of opposite chirality, where $a, \dot{a}=1, \ldots, 2^{d-1}$. The $\Gamma_{A}$ and $\Gamma_{\star}$ matrices have the form

$$
\left(\Gamma_{A}\right)_{\alpha}{ }^{\beta}=\left(\begin{array}{cc}
0 & \left(\Gamma_{A}\right)_{a}{ }^{b} \\
\left(\Gamma_{A}\right)_{\dot{a}}{ }^{b} & 0
\end{array}\right), \quad\left(\Gamma_{\star}\right)_{\alpha}{ }^{\beta}=\left(\begin{array}{cc}
\left(\mathbb{1}_{2^{d-1}}\right)_{a}{ }^{b} & 0 \\
0 & -\left(\mathbb{1}_{2^{d-1}}\right)_{\dot{a}}^{b}
\end{array}\right)
$$

In the Weyl basis the charge conjugation matrix is given by

$$
d \text { odd : } \quad C^{\alpha \beta}=\left(\begin{array}{cc}
0 & C^{a \dot{a}} \\
C^{\dot{a} a} & 0
\end{array}\right), \quad d \text { even }: C^{\alpha \beta}=\left(\begin{array}{cc}
C^{a b} & 0 \\
0 & C^{\dot{a} \dot{b}}
\end{array}\right) .
$$

Finally, a useful identity is

$$
\Gamma_{A_{1} \cdots A_{d}}=\frac{(-)^{d}}{d !} \epsilon_{A_{1} \cdots A_{d} B_{1} \cdots B_{d}} \Gamma_{*} \Gamma^{B_{d} \cdots B_{1}}
$$

To derive all the properties of the gamma matrices and the charge conjugation matrices that we have listed, it is convenient to work with an explicit representation of the gamma matrices. We first derive this representation in the Euclidean case, that is for $\mathrm{SO}(2 d)$, and we denote the corresponding gamma matrices with $\gamma_{A}$, to distinguish them from the ones of the maximally non-compact case. If $d=1$, the Clifford algebra is simply satisfied if $\gamma_{1}=\sigma_{1}$ and $\gamma_{2}=\sigma_{2}$, where $\sigma_{i}, i=1,2,3$ are the Pauli matrices, satisfying $\sigma_{i} \sigma_{j}=\delta_{i j}+i \epsilon_{i j k} \sigma_{k}$. To get the gamma matrices for $d=2$ one considers the tensor product of Pauli matrices and the two-dimensional identity matrix. One introduces $\gamma_{3}$ and $\gamma_{4}$, that start as $\gamma_{2}$, and to make these three matrices anticommuting one considers a tensor product with the three different Pauli matrices, that is $\gamma_{i+1}=\sigma_{2} \otimes \sigma_{i}$. The Clifford algebra is then satisfied if additionally $\gamma_{1}=\sigma_{1} \otimes \mathbb{1}_{2}$. This procedure can be induced to any $d$. Given the gamma matrices $\gamma_{A}, A=1, \ldots, 2 d$, in $2 d$ dimensions, that are made of tensor products of $d 2 \times 2$ matrices, one considers the matrices $\gamma_{A} \otimes \mathbb{1}_{2}$, for $A=1, \ldots, 2 d-1$, together with $\gamma_{2 d} \otimes \sigma_{1}$, 
$\gamma_{2 d} \otimes \sigma_{2}$ and $\gamma_{2 d} \otimes \sigma_{3}$. These matrices satisfy the Clifford algebra in $2(d+1)$ dimensions. This procedure leads to the following result

$$
\begin{aligned}
\gamma_{1} & =\sigma_{1} \otimes \mathbb{1}_{2} \otimes \mathbb{1}_{2} \otimes \mathbb{1}_{2} \otimes \mathbb{1}_{2} \otimes \ldots \\
\gamma_{2} & =\sigma_{2} \otimes \sigma_{1} \otimes \mathbb{1}_{2} \otimes \mathbb{1}_{2} \otimes \mathbb{1}_{2} \otimes \ldots \\
\gamma_{3} & =\sigma_{2} \otimes \sigma_{2} \otimes \mathbb{1}_{2} \otimes \mathbb{1}_{2} \otimes \mathbb{1}_{2} \otimes \ldots \\
\gamma_{4} & =\sigma_{2} \otimes \sigma_{3} \otimes \sigma_{1} \otimes \mathbb{1}_{2} \otimes \mathbb{1}_{2} \otimes \ldots \\
\gamma_{5} & =\sigma_{2} \otimes \sigma_{3} \otimes \sigma_{2} \otimes \mathbb{1}_{2} \otimes \mathbb{1}_{2} \otimes \ldots \\
\gamma_{6} & =\sigma_{2} \otimes \sigma_{3} \otimes \sigma_{3} \otimes \sigma_{1} \otimes \mathbb{1}_{2} \otimes \ldots \\
\gamma_{7} & =\sigma_{2} \otimes \sigma_{3} \otimes \sigma_{3} \otimes \sigma_{2} \otimes \mathbb{1}_{2} \otimes \ldots \\
\gamma_{8} & =\sigma_{2} \otimes \sigma_{3} \otimes \sigma_{3} \otimes \sigma_{3} \otimes \sigma_{1} \otimes \ldots \\
\gamma_{9} & =\sigma_{2} \otimes \sigma_{3} \otimes \sigma_{3} \otimes \sigma_{3} \otimes \sigma_{2} \otimes \ldots \\
\gamma_{10} & =\sigma_{2} \otimes \sigma_{3} \otimes \sigma_{3} \otimes \sigma_{3} \otimes \sigma_{3} \otimes \ldots \\
\vdots & =
\end{aligned}
$$

where it is understood that for $\mathrm{SO}(2 d)$ one uses $\gamma_{1}, \cdots, \gamma_{2 d}$ and takes in each expression the first $d$ factors of $2 \times 2$ matrices.

In this basis the matrices with odd index $\gamma_{2 n+1}$ are real and symmetric, while the matrices with even index $\gamma_{2 n}$ are imaginary and antisymmetric. To go to the $(d, d)$ signature, we define

$$
\Gamma_{2 n+1}=\gamma_{2 n+1} \quad \Gamma_{2 n}=i \gamma_{2 n}
$$

which implies that all the matrices $\Gamma_{n}$ are real, and are symmetric for $n$ odd and antisymmetric for $n$ even. The basis we have chosen is a Weyl basis, and the reader can verify that defining the chirality matrix as in (A.5) one obtains

$$
\Gamma_{\star}=\sigma_{3} \otimes \mathbb{1}_{2} \otimes \mathbb{1}_{2} \otimes \mathbb{1}_{2} \otimes \ldots
$$

which is indeed as in eq. (A.7).

If $d$ is even, one finds that the charge conjugation matrix

$$
C_{1}=\mathbb{1}_{2} \otimes \sigma_{2} \otimes \sigma_{1} \otimes \sigma_{2} \otimes \sigma_{1} \otimes \ldots \otimes \sigma_{1} \otimes \sigma_{2}
$$

satisfies eq. (A.3) with $\eta=-1$. One can also consider the charge conjugation matrix

$$
C_{2}=\sigma_{3} \otimes \sigma_{2} \otimes \sigma_{1} \otimes \sigma_{2} \otimes \sigma_{1} \otimes \ldots \otimes \sigma_{1} \otimes \sigma_{2}
$$

which satisfies eq. (A.3) with $\eta=+1$. Both these matrices have the same symmetry properties, and they are symmetric if $d / 2$ is even and antisymmetric if $d / 2$ is odd. Both these matrices commute with $\Gamma_{\star}$.

If $d$ is odd, one finds that the matrix

$$
C_{1}=\sigma_{2} \otimes \sigma_{1} \otimes \sigma_{2} \otimes \sigma_{1} \otimes \sigma_{2} \otimes \ldots \otimes \sigma_{1} \otimes \sigma_{2}
$$


satisfies eq. (A.3) with $\eta=+1$, and it is symmetric if $(d+1) / 2$ is even and antisymmetric if $(d+1) / 2$ is odd. The other solution of eq. (A.3) for $d$ odd is

$$
C_{2}=\sigma_{1} \otimes \sigma_{1} \otimes \sigma_{2} \otimes \sigma_{1} \otimes \sigma_{2} \otimes \ldots \otimes \sigma_{1} \otimes \sigma_{2}
$$

where in this case $\eta=-1$ and the symmetry properties are opposite to $C_{1}$. Both these matrices anticommute with $\Gamma_{\star}$. For any $d$, the product $C_{1} C_{2}$ is proportional to $\Gamma_{\star}$. All the properties we have derived here are listed in table 12 .

The $C$ matrices we have defined are hermitian, that is they are real if symmetric and imaginary if antisymmetric. Given that all the gamma matrices are real one can consider spinors with real components. When $C$ is real, given the real spinor $\lambda_{\alpha}$ one can then define the real spinor $\bar{\lambda}^{\alpha}$ from

$$
\bar{\lambda}^{\alpha}=\lambda_{\beta} C^{\beta \alpha} .
$$

Similarly, when $C$ is imaginary one can define

$$
\bar{\lambda}^{\alpha}=i \lambda_{\beta} C^{\beta \alpha}
$$

We now define the conjugate of $\Gamma_{A} \lambda$ (without loss of generality we can assume here that $C$ is real). One has

$$
{\overline{\left(\Gamma_{A} \lambda\right)}}^{\alpha}=\lambda_{\gamma}\left(\Gamma_{A}\right)_{\beta}^{\gamma} C^{\beta \alpha}=-\epsilon \lambda_{\gamma}\left(C \Gamma_{A}\right)^{\alpha \gamma}=-\eta\left(\bar{\lambda} \Gamma_{A}\right)^{\alpha} .
$$

The same relation occurs when $C$ is imaginary. In this paper we have made in all dimensions the choice for $C$ which gives $\eta=-1$. This implies that we have always used the relation

$$
{\overline{\left(\Gamma_{A} \lambda\right)}}^{\alpha}=\left(\bar{\lambda} \Gamma_{A}\right)^{\alpha} \text {. }
$$

This also implies that we have chosen $C$ to be imaginary for $d=2$ and $d=3$, while $C$ is real for $d=1, d=4$ and $d=5$, as can be deduced by looking at the values of $\epsilon$ corresponding to $\eta=-1$ in table 12 .

In this paper we have considered bilinears of $\mathrm{SO}(d, d)$ spinors. In particular, in section 5 we have counted the number of world-volume degrees of freedom of various brane effective actions by looking at the world-volume fields that appear in the WZ term. In order to perform this counting, one has to determine the number of different components of the $\mathrm{SO}(d, d)$ spinors that appear in a bilinear containing $m$ Gamma matrices with fixed vector $\mathrm{SO}(d, d)$ indices. We now want to show that to perform this computation one has to go to light-cone vector indices of the T-duality group. We denote these indices by $1 \pm, 2 \pm, \ldots, d \pm$. If $m=1$, we therefore take the single gamma matrix to be along such directions. If $m=2$, we will see that one has to take the product of two Gamma matrices in the directions $n_{1} \pm, n_{2} \pm$, with $n_{1} \neq n_{2}$. These directions form a conjugacy class inside the representation corresponding to two antisymmetric $\mathrm{SO}(d, d)$ indices. Similarly, for $m=3$ and $m=4$ one has to take the the products of Gamma matrices in the directions $n_{1} \pm, n_{2} \pm, \ldots, n_{m} \pm$, with $n_{1}, n_{2}, \ldots, n_{m}$ all different. For any $m$, the dimension of this conjugacy class is

$$
2^{m}\left(\begin{array}{c}
d \\
m
\end{array}\right)
$$


and it is equal to the dimension of the corresponding representation, $\left(\begin{array}{c}2 d \\ m\end{array}\right)$, only for $m=0$ and $m=1$. The dimension of the conjugacy class for $m=2, m=3$ and $m=4$ is given in table 9 for $d=2, \ldots, 5$.

The products of Gamma matrices in the conjugacy classes defined above all have the property of being nilpotent. We now want to show that spinor bilinears formed out of these matrices relate $2^{d-m}$ spinor components, with $m \leq d$, that is $2^{d-m-1}$ of one chirality and $2^{d-m-1}$ of the opposite chirality. As we will see, the case $m=d$ is special because a nilpotent product of $m$ light-cone Gamma matrices maps a generic spinor to a single component of a given chirality. The case $m>d$ is related to the previous case by using the epsilon symbol, but we are not interested in this case because it never gives rise to WZ terms that satisfy our criteria.

To show that the nilpotent product of $m$ light-cone Gamma matrices, $m \leq d$, maps a $2^{d}$-component spinor to $2^{d-m}$ components, we make a specific choice of basis and we show that in this basis the light-cone matrices have $2^{d-m}$ non-vanishing entries. For any $d$ one can take the light-cone basis and define the nilpotent matrices

$$
\begin{aligned}
& \Gamma_{1 \pm}=\frac{1}{2}\left(\Gamma_{1} \pm \Gamma_{2 d}\right) \\
& \Gamma_{n \pm}=\frac{1}{2}\left(\Gamma_{2 n-2} \pm \Gamma_{2 n-1}\right) \quad n=2, \ldots, d,
\end{aligned}
$$

where the standard Gamma matrices are those given in (A.10) and (A.11). Before discussing the general case, we first consider the cases $d=1$ and $d=2$ explicitly.

For $d=1$ the Gamma matrices are $2 \times 2$ matrices, and eq. (A.22) gives

$$
\Gamma_{1+}=\left(\begin{array}{cc}
0 & 1 \\
0 & 0
\end{array}\right) \quad \Gamma_{1-}=\left(\begin{array}{ll}
0 & 0 \\
1 & 0
\end{array}\right) \text {. }
$$

One can see that, given a generic 2-component spinor, each of these matrices maps to a given chirality. This is obvious, because these two matrices are nothing but $\Gamma_{A} \pm \epsilon_{A B} \Gamma^{B}$.

In the $d=2$ case the matrices are $4 \times 4$, and using eq. (A.22) one obtains

$$
\begin{aligned}
& \Gamma_{1+}=\left(\begin{array}{ccc} 
& 1 & 0 \\
& 0 & 0 \\
0 & 0 & \\
0 & 1 &
\end{array}\right) \quad \Gamma_{1-}=\left(\begin{array}{lll} 
& 0 & 0 \\
& 0 & 1 \\
1 & 0 & \\
0 & 0 &
\end{array}\right) \\
& \Gamma_{2+}=\left(\begin{array}{rrrr} 
& & 0 & 0 \\
& 1 & 0 \\
0 & 0 & \\
-1 & 0 &
\end{array}\right) \quad \Gamma_{2-}=\left(\begin{array}{rrr} 
& 0 & 1 \\
& 0 & 0 \\
0 & -1 & \\
0 & 0 &
\end{array}\right) \text {, }
\end{aligned}
$$

where here and in the following the blocks that are left blank have all zero entries. One can see that each of these matrices maps a generic 4-component spinor to one component of each chirality. We next consider $m=2$, that is the antisymmetric product of two Gamma matrices. Given the 6 independent $\Gamma_{A B}$ matrices, one can choose in light-cone coordinates 
the basis $\Gamma_{1 \pm} \Gamma_{2 \pm},\left(\Gamma_{1+} \Gamma_{1-}-\Gamma_{1-} \Gamma_{1+}\right)$ and $\left(\Gamma_{2+} \Gamma_{2-}-\Gamma_{2-} \Gamma_{2+}\right)$, where only the first four matrices belong to the nilpotent conjugacy class. In particular, one has

$$
\Gamma_{1+} \Gamma_{2+}=\left(\begin{array}{ccc}
0 & 0 & \\
0 & 0 & \\
& 0 & 0 \\
& 1 & 0
\end{array}\right)
$$

which has only one non-zero entry, and thus maps a spinor to one component of a given chirality. The same applies to the other three matrices obtained by taking the other combinations of pluses and minuses, while the reader can verify that this does not apply to (linear combinations of) the other two matrices $\left(\Gamma_{1+} \Gamma_{1-}-\Gamma_{1-} \Gamma_{1+}\right)$ and $\left(\Gamma_{2+} \Gamma_{2-}-\Gamma_{2-} \Gamma_{2+}\right)$.

We now briefly consider the general case $d>2$. We explicitly write the matrices $\Gamma_{2 \pm}$ and $\Gamma_{3 \pm}$. For $\Gamma_{2 \pm}$ using (A.10) and (A.11) one gets



where $\mathbb{O}$ and $\mathbb{1}$ are the $2^{d-2} \times 2^{d-2}$ zero and identity matrices. Similarly, for $\Gamma_{3 \pm}$ one gets

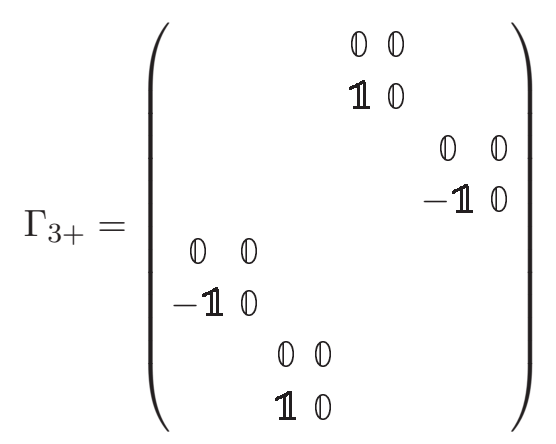

and

$$
\Gamma_{3-}=\left(\begin{array}{ccccccc} 
& & & 0 & \mathbb{1} & & \\
& & 0 & 0 & & \\
& & & & 0 & -\mathbb{1} \\
& & & & 0 & 0 \\
& & & & & 0 \\
0 & -\mathbb{1} & & & & & \\
0 & 0 & & & & & \\
& & 0 & \mathbb{1} & & & \\
& 0 & 0 & & & &
\end{array}\right)
$$

where here $\mathbb{0}$ and $\mathbb{1}$ are the $2^{d-3} \times 2^{d-3}$ zero and identity matrices. One can see that each of these matrices halves the spinor components of each chirality. The product of the two 
Gamma matrices $\Gamma_{2+}$ and $\Gamma_{3+}$ is

$$
\Gamma_{2+} \Gamma_{3+}=\left(\begin{array}{cccccc} 
& 0 & 0 & & & \\
& 0 & 0 & & \\
0 & 0 & & & & \\
-1 & 0 & & & & \\
& & & & 0 & 0 \\
& & & & 0 & 0 \\
& & 0 & 0 & \\
& & -1 & 0 &
\end{array}\right),
$$

where again $\mathbb{O}$ and $\mathbb{1}$ are the $2^{d-3} \times 2^{d-3}$ zero and identity matrices and thus this matrix maps a $2^{d}$-component spinor to $2^{d-3}$ components for each chirality. The reader can verify that the same applies to the other three combinations of pluses and minuses, while it does not apply to $\left(\Gamma_{2+} \Gamma_{2-}-\Gamma_{2-} \Gamma_{2+}\right)$, as well as to $\left(\Gamma_{3+} \Gamma_{3-}-\Gamma_{3-} \Gamma_{3+}\right)$.

We consider explicitly the case $d=3$, for which the entries in eq. (A.29) are numbers. We then compute $\Gamma_{1+}$ from eq. (A.22), that is

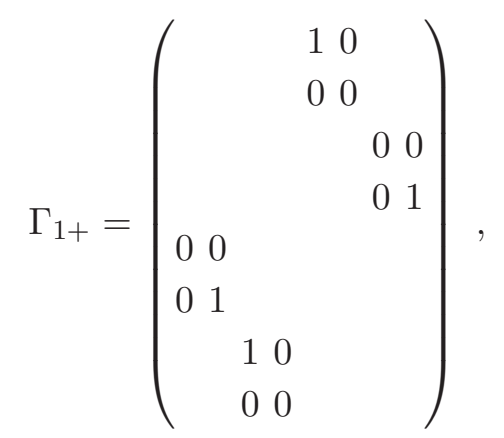

and we finally consider the product of the three light-cone Gamma matrices

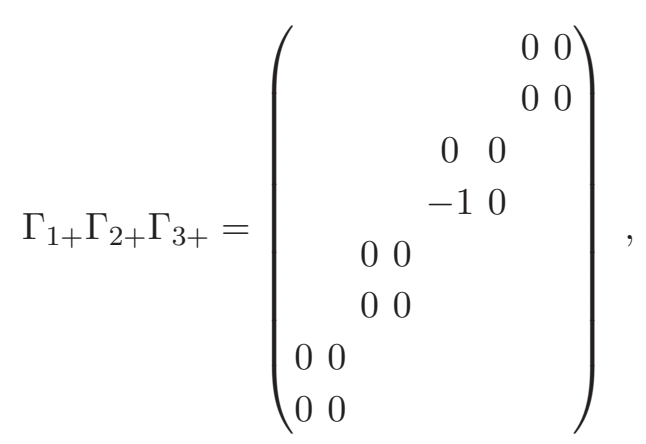

which as expected has only one non-zero entry, and thus maps an 8-component spinor to a single component of a given chirality.

A careful analysis of the Gamma matrices defined in eqs. (A.10) and (A.11) and the light-cone Gamma matrices of eq. (A.22) should convince the reader that the result is completely general and applies to all cases.

Open Access. This article is distributed under the terms of the Creative Commons Attribution Noncommercial License which permits any noncommercial use, distribution, and reproduction in any medium, provided the original author(s) and source are credited. 


\section{References}

[1] E. Bergshoeff, M. de Roo, M.B. Green, G. Papadopoulos and P.K. Townsend, Duality of Type II 7-branes and 8-branes, Nucl. Phys. B 470 (1996) 113 [hep-th/9601150] [SPIRES].

[2] E.A. Bergshoeff, M. de Roo, S.F. Kerstan and F. Riccioni, IIB Supergravity Revisited, JHEP 08 (2005) 098 [hep-th/0506013] [SPIRES].

[3] E.A. Bergshoeff, M. de Roo, S.F. Kerstan, T. Ortín and F. Riccioni, IIA ten-forms and the gauge algebras of maximal supergravity theories, JHEP 07 (2006) 018 [hep-th/0602280] [SPIRES].

[4] E.A. Bergshoeff, J. Hartong, P.S. Howe, T. Ortín and F. Riccioni, IIA/ IIB Supergravity and Ten-forms, JHEP 05 (2010) 061 [arXiv:1004.1348] [SPIRES].

[5] L.J. Romans, Massive $N=2$ a Supergravity in Ten-Dimensions, Phys. Lett. B 169 (1986) 374 [SPIRES].

[6] B. de Wit, H. Nicolai and H. Samtleben, Gauged Supergravities, Tensor Hierarchies and M-theory, JHEP 02 (2008) 044 [arXiv:0801.1294] [SPIRES].

[7] F. Riccioni and P.C. West, The $E_{11}$ origin of all maximal supergravities, JHEP 07 (2007) 063 [arXiv:0705.0752] [SPIRES].

[8] E.A. Bergshoeff, I. De Baetselier and T.A. Nutma, $E_{11}$ and the embedding tensor, JHEP 09 (2007) 047 [arXiv:0705.1304] [SPIRES].

[9] P.C. West, $E_{11}$ and M-theory, Class. Quant. Grav. 18 (2001) 4443 [hep-th/0104081] [SPIRES].

[10] E.A. Bergshoeff and F. Riccioni, D-Brane Wess-Zumino Terms and U-duality, JHEP 11 (2010) 139 [arXiv:1009.4657] [SPIRES].

[11] P.P. Cook and P.C. West, Charge multiplets and masses for $E_{11}$, JHEP 11 (2008) 091 [arXiv:0805.4451] [SPIRES].

[12] P.P. Cook, Exotic $E_{11}$ branes as composite gravitational solutions, Class. Quant. Grav. 26 (2009) 235023 [arXiv:0908.0485] [SPIRES].

[13] J.H. Schwarz, An SL(2,Z) multiplet of type IIB superstrings, Phys. Lett. B 360 (1995) 13 [hep-th/9508143] [SPIRES].

[14] M. Aganagic, J. Park, C. Popescu and J.H. Schwarz, Dual D-brane actions, Nucl. Phys. B 496 (1997) 215 [hep-th/9702133] [SPIRES].

[15] P.K. Townsend, Membrane tension and manifest IIB S-duality, Phys. Lett. B 409 (1997) 131 [hep-th/9705160] [SPIRES].

[16] M. Cederwall and A. Westerberg, World-volume fields, $\operatorname{SL}(2, Z)$ and duality: The type IIB 3-brane, JHEP 02 (1998) 004 [hep-th/9710007] [SPIRES].

[17] A. Westerberg and N. Wyllard, Towards a manifestly $\mathrm{SL}(2, Z)$-covariant action for the type IIB (p,q) super-five-branes, JHEP 06 (1999) 006 [hep-th/9905019] [SPIRES].

[18] E.A. Bergshoeff, M. de Roo, S.F. Kerstan, T. Ortín and F. Riccioni, SL(2, R)-invariant IIB brane actions, JHEP 02 (2007) 007 [hep-th/0611036] [SPIRES].

[19] C.M. Hull, A geometry for non-geometric string backgrounds, JHEP 10 (2005) 065 [hep-th/0406102] [SPIRES]. 
[20] C.M. Hull, Doubled geometry and T-folds, JHEP 07 (2007) 080 [hep-th/0605149] [SPIRES].

[21] C.M. Hull and R.A. Reid-Edwards, Gauge Symmetry, T-duality and Doubled Geometry, JHEP 08 (2008) 043 [arXiv:0711.4818] [SPIRES].

[22] P. Meessen and T. Ortín, An $\mathrm{Sl}(2, Z)$ multiplet of nine-dimensional type-II supergravity theories, Nucl. Phys. B 541 (1999) 195 [hep-th/9806120] [SPIRES].

[23] G. Dall'Agata, K. Lechner and M. Tonin, $D=10, N=I I B$ supergravity: Lorentz-invariant actions and duality, JHEP 07 (1998) 017 [hep-th/9806140] [SPIRES].

[24] E.A. Bergshoeff, J. Hartong, T. Ortín and D. Roest, Seven-branes and supersymmetry, JHEP 02 (2007) 003 [hep-th/0612072] [SPIRES].

[25] E.A. Bergshoeff, M. de Roo, S.F. Kerstan, T. Ortín and F. Riccioni, IIB nine-branes, JHEP 06 (2006) 006 [hep-th/0601128] [SPIRES].

[26] R. Slansky, Group Theory for Unified Model Building, Phys. Rept. 79 (1981) 1 [SPIRES].

[27] P.K. Townsend, M-theory from its superalgebra, hep-th/9712004 [SPIRES].

[28] E. Bergshoeff, B. Janssen and T. Ortín, Kaluza-Klein monopoles and gauged $\sigma$-models, Phys. Lett. B 410 (1997) 131 [hep-th/9706117] [SPIRES].

[29] I. Schnakenburg and P.C. West, Kac-Moody symmetries of IIB supergravity, Phys. Lett. B 517 (2001) 421 [hep-th/0107181] [SPIRES].

[30] A. Kleinschmidt, I. Schnakenburg and P.C. West, Very-extended Kac-Moody algebras and their interpretation at low levels, Class. Quant. Grav. 21 (2004) 2493 [hep-th/0309198] [SPIRES].

[31] M.R. Gaberdiel, D.I. Olive and P.C. West, A class of Lorentzian Kac-Moody algebras, Nucl. Phys. B 645 (2002) 403 [hep-th/0205068] [SPIRES].

[32] T. Damour, M. Henneaux and H. Nicolai, $E_{10}$ and a 'small tension expansion' of M-theory, Phys. Rev. Lett. 89 (2002) 221601 [hep-th/0207267] [SPIRES].

[33] P.C. West, Very extended $E_{8}$ and $A_{8}$ at low levels, gravity and supergravity, Class. Quant. Grav. 20 (2003) 2393 [hep-th/0212291] [SPIRES].

[34] F. Riccioni and P.C. West, Dual fields and $E_{11}$, Phys. Lett. B 645 (2007) 286 [hep-th/0612001] [SPIRES].

[35] V.G. Kac, Infinite dimensional Lie algebras, Cambridge Univ. Pr., Cambridge U.K. (1990) [SPIRES].

[36] P.C. West, The IIA, IIB and eleven dimensional theories and their common $E_{11}$ origin, Nucl. Phys. B 693 (2004) 76 [hep-th/0402140] [SPIRES].

[37] E.A. Bergshoeff, M. de Roo, S.F. Kerstan, A. Kleinschmidt and F. Riccioni, Dual Gravity and Matter, Gen. Rel. Grav. 41 (2009) 39 [arXiv:0803.1963] [SPIRES].

[38] A. Van Proeyen, Tools for supersymmetry, hep-th/9910030 [SPIRES].

[39] F. Riccioni, Low-energy structure of six-dimensional open-string vacua, hep-th/0203157 [SPIRES]. 